\title{
Ricerche sulla varietà cubica generale dello spazio a quattro dimensioni e sopra i suoi spazi pluritangenti.
}

\author{
(Di Givo Fano, a Torino.)
}

In questa Memoria vengono trattate alcune questioni di carattere projettivo riguardanti la varietà cubica generale (priva di punti doppi) dello spazio a quattro dimensioni. Fra altro, vengono completamente determinati gli spazi che sono tangenti a questa varietà in 2, 304 punti diversi, nonchè le proprietà e $\mathrm{i}$ caratteri di alcuni enti a cui quegli spazi danno luogo. Un procedimento per la costruzione degli spazi pluritangenti di questa stessa varietà è stato dato parecchi anni or sono dal sig. Enriques (*), il quale ne ha anche dedotta la valutazione di alcuni dei caratteri sopra accennati; lo stesso procedimento, insieme con altri; trova pure applicazione in questa Memoria, ma quei caratteri risultano ora modificati (si vegga in proposito quanto è detto in una nota al $\mathrm{n}^{\circ} 8$ ). $\mathrm{E}$, in conseguenza, risultano pure modificati alcuni caratteri delle varietà cubiche e loro Hessiane, che si trovano nell'ultima parte di un lavoro, di poco posteriore, del sig. Ascrone $\left(^{* *}\right)$.

1. Sia $V^{3}$ (o brevemente $V$ ) una varietà cubica a trè dimensioni dello spazio $S_{4}$, priva di punti doppi. Ogni punto di questo spozio avrà rispetto ad essa una certa quadrica polare; e tutte queste quadriche formeranno un sistema lineare $\infty^{4}$, privo di punti basi. $\mathrm{Vi}$ sarà in particolare una triplice infinità di punti le cui quadriche polari hanno un punto doppio, cioè sono

(*) Sugli spazi pluritangenti delle varieta cubiche generali appartenenti allo spazio a quattro dimensioni (Giorn. di matem., vol. 31 (1893); p. $31-35$ ).

(*)) Sulla Iressiana di una varieti nello spazio a quattro dimensioni (ibid., p. 210-17). 
252 Fano: Ricerche sulla varietà cubica generale dello spazio

coni. Il luogo di questi punti è la varielà Hessiana di $V$, la quale è del $5 .^{\circ}$ ordine, ed è anche in pari tempo il luogo dei vertici di quei coni quadrici. Anzi - analogamente a quanto avviene nel piano e nello spazio ordinario (*) - se la quadrica polare di un punto $X$ è un cono di vertice $Y$, a sua volta $Y$ avrà per quadrica polare un cono di vertice $X$; i due punti $X$ e $Y$ si diranno allora "punti corrispondenti " della varietà Hessiana, e saranno reciproci rispetto a tutte le quadriche del sistema polare (e viceversa).

La varietà Hessiana di $V$ ha una curva doppia di ordine $20 *^{* *}$ ), luogo dei punti le cui quadriche polari sono coni di 2." specie. Ciascuno di questi punti ha, sulla Hessiana, infiniti corrispondenti: tutti quelli della retta asse del suo cono polare; ed è percio vertice di tutto un fascio di coni del sistema polare.

La varietà Hessiana incontra $V$ secondo una superficie del $15 .^{\circ}$ ordine, che si può chiamare la "superficie parabolica" di $V$. Gli spazi $S_{3}$ tangenti a $V$ nei punti di questa superficie hanno la proprietà caratteristica di incontrare $V$ secondo superficie cubiche con punto doppio biplanare; e gli spazi tangenti a $V$ nei $20.3=60$ punti comuni a questa varietà e alla curva doppia della varietà Hessiana incontrano $T$ secondo superficie con punto doppio uniplanare.

Lá varietà $V$ è di classe 24 , ossia per un piano generico passano 24 spazi ad essa tangenti. I punti di contatto di questi spazi sono le intersezioni di $V$ colla curva di $8 .^{\circ}$ ordine che è base della rete formata dalle quadriche polari dei punti del piano considerato.

Non insistiamo ulteriormente sopra queste proprietà, perchè esse possono riguardarsi come generalmente note. $\mathrm{E}$ ci riserviamo pure di considerare in seguito come note, quando occorra farne uso, tutte le proprietà fondamentali della polarità rispetto alla varietà $V$.

2. Una retta generica di $S_{4}$ è contenuta in una semplice infinità di quadriche del sistema polare determinato da $V$, formanti un fascio. Vi sono

(i) Cfr. ad es, SAlmox-Frodur, Analytische Theorie der höheren ebenen Curven, p. 193-94; e: Analytische Geometrie des Raumes, II, p. 377-78.

(*) Questa curva si rappresenta analiticamente scrivendo che il determinante Hessiano di $V$, che è simmetrico del $5 .^{\circ}$ ordine, ha la caratteristica tre; e di qui segue appunto la determinazione dell'ordine della curva stessa. Per la formola generale applicabile a questo caso V. C. SEGRE: Gli ordini delle varieta che amullano i determinanti dei vari gradi estratti di una matrice (Rend. Lincei (5), IX, 1900). 
però delle rette particolari, ciascuna delle quali appartiene a un'intera rete di queste quadriche; ad esse daremo il nome di rette "specialin $\left(^{*}\right)$. Sopra una retta speciale le $\infty^{4}$ quadriche del sistema polare segheranno soltanto una semplice infinità, vale a dire un'involuzione di coppie di punti; e ciascuna di queste coppie farà parte di un gruppo $G_{16}$ di 16 punti, base di un sistema lineare $\infty^{3}$ di quadriche contenuto nel sistema polare complessivo $\infty$. Viceversa, ogni retta la quale congiunga due punti di un tale gruppo $G_{16}$ imporrà una sola condizione ulteriore a una delle $\infty^{3}$ quadriche passanti per questo $G_{86}$ la quale debba contenerla per intero; essa apparterrà perciò a una doppia infinità di queste quadriche - sarà dunque una retta " speciale " - e conterrà, oltre a quella prima coppia di punti, anche $\infty^{1}$ altre coppie analoghe, appartenenti a altrettanti diversi gruppi $G_{16}$. E poichè ogni punto di $S_{4}$ appartiene a uno e un solo gruppo $G_{16}$, cosi per quel punto passeranno precisamente 15 rette speciali (quelle che lo congiungono ai rimanenti punti del medesimo gruppo $G_{16}$ ); e le rette speciali di tutto lo spazio $S_{4}$ saranno in numero di $\infty^{3}$.

Le $\infty^{2}$ quadriche del sistema polare che passano per una retta speciale avranno a comune, oltre questa retta, una curva di $7 .^{\circ}$ ordine e genere 3 avente tale retta per trisecante. Questa curva la chiameremo brevemente la “ $C_{3}^{7}$ residua" di quella retta.

Nello spazio $S_{4}$ (anzi in ogni spazio lineare) le rette contenute nelle $\infty^{2}$ quadriche di una rete formano un complesso cubico (**). Infatti per ogni punto dello spazio passano $\infty^{t}$ quadriche della rete, formanti un fascio; le rette contenute in queste $\infty^{1}$ quadriche sono le corde della varietà $q^{4}$ base del fascio; e, fra queste rette, quelle che escono dal punto considerato saranno le generatrici del cono cubico che da questo medesimo punto proietta la $\varsigma^{4}$. - Ora nel sistema polare $\propto^{4}$ determinato dalla nostra varietà cubica $Y$ sono contenute $\infty^{6}$ reti di quadriche, le quali individuano altrettanti complessi cubici di rette; e si vede immediatamente che il sistema $\infty^{3}$ delle rette speciali è l'intersezione completa di questi $\infty^{6}$ complessi cubici (***).

(*) Enriques, 1. c. p. 32.

(*) Ossia una varietà rappresentata da un'unica equazione di $3^{\circ}$ grado fra le ordinarie coordinate di retta $\gamma_{i k}=\left(x_{i} y_{k}\right)$.

(**) Qualunque sistema lineare $\infty^{4}$ di quadriche dello spazio $S_{4}$ (anche se non è pre. cisamente il sistema delle quadriche polari vispetto a una varieti cubica) da egualmente origine a un sistema $\infty^{3}$ di rette «peciali》, contenuto in $\infty^{n}$ complessi cubici. Il sistema 
Gli spazi $S_{3}$ polari dei punti di una retta speciale (rispetto alla varietà $V$ ) formano un fascio, avendo a comune l"intero piano luogo dei punti le cui quadriche polari passano per quella retta; e ogni $S_{3}$ di questo fascio è spazio polare di due punti di quella retta. (Invece gli $S_{3}$ polari dei punti di una retta generica hanno a comune soltanto una retta, e inviluppano un cono quadrico di 2." specie avente quest'ultima retta per asse).

3. I diversi gruppi $G_{16}$ considerati al n. ${ }^{\circ}$ prec. formano nello spazio $S_{4}$ un'involuzione $I_{16}$; e ciascuno di essi si compone dei poli di un medesimo spazio $S_{3}$. Quando fra questi 16 poli di uno spazio $F_{3}$ due (almeno) coincidono in un punto $P$, il sistema lineare $\infty^{3}$ formato dalle quadriche polari dei punti di questo $S_{3}$, per il quale quel $G_{16}$ è gruppo base, avrà in $P$ (almeno) una tangente fissa; e allora, imponendo come tangenti in $P$ tre altre rette arbitrarie uscenti da questo punto, si vede, che vi sarà nel detto sistema $\infty^{3}$ una quadrica avente in $P$ un punto doppio, vale a dire un cono di vertice $P$ : sarà dunque $P$ un punto della varietà Hessiana di $V$ (e viceversa).

Ora sopra ogni retta sptciale $r$ vi è un'involuzione di coppie di punti contenute rispett. in altrettanti gruppi $G_{16}$. Perciò ciascuno dei due punti doppi $M, N$ di questa involuzione coinciderà con uno dei 15 suoi coniugati nella $I_{16}$ (e le $\infty^{3}$ quadriche del sistema polare che passano per questo punto saranno tutte ivi tangenti alla retta $r$ ): sicchè i due punti $M$ e $N$ staranno sulla varietà Hessiana di $V$. E anzi, poichè questi due punti sono evidentemente reciproci rispetto a tutte le quadriche del sistema polare, essi saranno altresi "punti corrispondenti" della Ilessiana (n." 1). - Le rimanenti tre intersezioni di $r$ colla varietà Hessiana (che è del $5 .^{\circ}$ ordine) saranno $\mathrm{i}$ tre punti comuni a $\dot{r}$ stessa e alla sua $C_{3}^{7}$ residua: poichè in ognuno di questi punti le $\infty^{2}$ quadriche del sistema polare passanti per $r$, e quindi anche per la $C_{3}^{7}$, hanno un piano tangente fisso (il piano determinato da $r$ e dalla tangente alla $C_{3}^{z}$ ), e perciò tutte le $\infty^{3}$ quadriche del sistema polare che passano per quel punto vi avranno ancora una tangente fissa.

analogo $\left(\infty^{2}\right)$ di rette nello spazio ordinario (di ordine 7 e classe 3 ) é stato studiato dal Sig. REYe (Geometrie der Lage, 3te Aufl., III, p. 140 e seg.), e fu incontrato anche da me in altre ricerche (Nuove vicerche sulle congruenze di rette del $30^{\circ}$ ordine prive di linea singolare, Mem. Acc. Torino, ser. II, t. L, 1901 ; \$ 14). 
Le cinque intersezioni di una retta speciale colla varietà Hessiana di $V$ sono $i$ due punti doppi dell'involuzione segata sopra questa retta dal sistema polare di quadriche, e le tre intersezioni della medesima retta colla sua $C_{3}^{7}$ residua.

Questi cinque punti sono in generale distinti. E la retta $r$ sarà tangento alla varietà Hessiana in uno dei due punti $M, N$ sopra considerati soltanto quando la sua $C_{3}^{*}$ residua passa per questo punto. Ci conviene però di dare a questa condizione una forma diversa.

4. Per una proprietà nota delle curve piane e superficie del terzo ordine (*), la quale si estende immediatamente alle variełà cubiche di uno spazio qualsiasi, due punti corrispondenti della Hessiana ài una varietà cubica $V$ sono sempre tali che lo spazio tangente alla Hessiana medesima in uno di essi è polare dell'altro rispetto alla variet̀̀ $V$. Ora, se la retta speciale $r$ considerata al $\mathrm{n}^{\circ}$ prec. è tangente alla Hessiana p. e. nel punto $M$, essa dovrà stare nello spazio tangente alla Hessiana in questo medesimo punto, il quale non è altro che to spazio polare di $N$ rispetto a $V$; questo spazio passerà allora anche per il punto $N$, e questo punto starà perciò sulla varietà cubica $V$ (e viceversa). La quadrica polare di $N$, che è un cono di vertice $M$, dovrà allora passare per $N$, ossia avrà come generatrice la retta $r \equiv M N$; e questa retta sarà perciò una tangente tripunta di $V$ nel punto $N$.

Una retta speciale $r \equiv M N$ è tangente alla varietà Hessiana in uno dei due punti $M, N$ sempre e solo quando l'altro di questi due punti sta sulla varietà $V$. La retta $\mathrm{r}$ è allora tangente tripunta di $V$ in questo secondo punto.

Più particolarmente, perchè la retta speciale $r \equiv M N$ sia bitangente della Hessiana (nei due punti $M, N$ ) sarà necessario e sufficiente ch'essa sia in entrambi questi punti tangente tripunta (almeno) della $V$; e cio avviene soltanto quando essa è contenuta in quest'ultima varietà.

Le (sole) rette speciali contenute nella varietà $V$ sono bitangenti della varietà Hessiana; e la toccano precisamente nei punti doppi delle involuzioni segate su di esse dalle quadriche del sistema polare $\left.{ }^{* *}\right)$.

$(*)$ Salmox-Fiedler, op. cit,; v. rispett, p. 196 e p. 378.

(**) Questa proprieta è l'immediata estensione di quella che riguarda il comportamento delle rette contenute in una superficie cubica rispetto alla linea parabolica di questa superficie (SaLion-Fiedilar, op. cit., p. 399). 
Spazi bitangentri.

5. Quando una retta speciale è contenuta nella varietà $V$, lo spazio $S_{3}$ polare di un suo punto qualunque e che è pure polare di un secondo punto della stessa retta (coniugato del primo nell'involuzione $I_{16}$ ) sarà tangente a $V$ in entrambi questi punti; sarà dunque uno spazio bitangente. $\mathrm{E}$ viceversa, se la varietà $V$ è toccata da uno spazio $S_{3}$ in due diversi punti $A$ e $A^{\prime}$, anzitutto la retta $A A^{\prime}$ sarà una retta speciale, perchè congiunge due fra i 16 poli di quello spazio; e essa apparterrà altresì alla varietà $V$, essendo tangente a questa in entrambi quei punti. Gli $\infty^{1}$ spazi tangenti a $V$ nei punti di una tale retta $p$ saranno tutti spazi bitangenti, col secondo punto di contatto sopra questa stessa retta; essi formeranno un fascio $\left(n .^{\circ} 2\right)$, e avranno a comune un piano $\pi$ che dovrà passare per $p$ e sarà tangente a $V$ in ogni punto di questa retta. Questo piano incontrerà $V$ secondo la retta $p$ contata due volte, più una retta ulteriore (che si potrì chiamare la " coniugata $n$ di $p$ ), in generale distinta da $p$ stessa. E poichè la varietà $V$ ha un numero doppiamente infinito di spazi bitangenti, così le rette speciali $p$ contenute in $V$ saranno in numero semplicemente infinito e formeranno una rigata $\Sigma$, Juogo dei punti di contatto di quegli spazi; lungo ognuna di queste $\infty^{1}$ rette $p$ la varietà $V$ ammetterà un piano tangente fisso $\pi$, e gli $\boldsymbol{\alpha}^{2}$ spazi bitangenti di $V$ si distribuiranno negli $\alpha^{\prime}$ fasci che hanno per assi questi piani $\pi$.

Ognuna delle $\infty^{1}$ rette speciali $p$ avrà la proprietà di contare come due (almeno) fra le sei rette della varietà $V$ che escono da uno qualunque dei suoi punti (poichè lo spazio $S_{3}$ tangente a $V$ in questo punto è tangente anche in un secondo punto di quella medesima retta; e sopra una superficie cubica di $S_{3}$ con due punti doppi la retta che congiunge questi punti conta sempre come due almeno fra le sei rette della superficie che escono sia dall'uno che dall'altro di essi). Viceversa, se fra le sei rette di $V$ che escono da un punto $X$ due (almeno) coincidono con una certa retta $x$, lo spazio $S_{s}$ tangente a $V$ in $X$ dovià toccare questa varietà anche in un secondo punto di quella stessa retta (in generale distinto dal primo, ma che potrebbe tuttavia essergli infinitamente vicino); quello spazio sarà perciò uno spazio bitangente, e $x$ sarà una retta speciale. La superficie rigata $\Sigma$ sarà perciò anche, 
in pari tempo, il luogo di quei punti di $V$ pei quali coincidono due almeno delle sei rette di questa varietà che ne escono.

6. Dico ora che la rigata $\Sigma$ testè considerata è una sviluppabile.

Ricordiamo perciò che nella varietà cubica generale $V$ è contenuta una doppia infinità di rette; e a ognuna di queste ne sono infinitamente vicine, sopra $V$ stessa, $\infty$ altre. Una retta non speciale contenuta in $V$ è tale che tutte quelle ad essa infinitamente vicine sono sghembe rispetto ad essa; poichè da nessun punto dj quella retta esce una seconda retta di $V$ infinitamente vicina alla prima. - Sia invece $p$ una retta speciale contenuta in $V$; e sia ז. il piano tangente a $V$ lungo di essa, e $P$ un punto qualunque di $p$. Si consideri poi sopra $V$, ma fuori della superficie $\Sigma$, un punto $P^{\prime}$ convenientemente prossimo a $P$; e questo punto si faccia tendere alla posizione $P$, muovendosi sopra $V$ con continuità. Allora due fra le 6 rette di $V$ uscenti dal punto $P^{\prime}$ tenderanno alla posizione $p$; e il fascio da esse individuato tenderà al fascio $P(\pi)$. Questo è dunque un fascio (o varietà lineare $\left.\propto^{1}\right)$ di rette tangente nell'elemento $p$ al sistema $\infty^{2}$ formato da tutte le rette contenute in $V$; e poichè $P$ è un punto arbitrario della retta $p$, così la varietà lineare $\alpha^{2}$ di rette tangente a quel medesimo sistema nell'elemento $p$ dovrà contenere tutti gli $\infty^{1}$ fasci $P(\pi)$, e non sarà dunque altro che il piano rigato $\pi$. Siccome le rette di questo piano sono tutte incidenti alla $p$, così potremo dire che una retta speciale contenuta nella varietà $V$ è invece incidente a tutte quelle ad essa infinitamente vicine: queste $\infty^{1}$ rette consecutive a $p$ sopra $V$ appartengono ai singoli fasci $P(\pi)$, ciò̀ escono rispett. dai singoli punti di $p$ e stanno tutte nel piano $\pi\left(^{*}\right)$.

Ciò premesso, è chiaro che in una rigata formata da rette contenute in $V$ una generatrice qualsiasi sarà generatrice singolare, ossia incidente alla consecutiva, sempre e solo quando essa sia retta speciale (nel senso di cui

*) Questo fatto è d'accordo con un'osservazione fatta dal Sir. Kukin (Ueber Flächen 3ter Ordnung, Math. Ann. VI, p. 596) che una superficie cubica di $S_{3}$ sulla quale due rette si facciano avvicinare indefinitamente acquista al limite, sopra queste rette, uno o due punti doppi, secondo che tali rette al limite sono sghembe, oppure incidenti. Ora lo spazio tangente alla varieta $V$ in un punto di una retta speciale tocca quella varieta anche in un secondo punto di questa retta, e incontra perció $V$ secondo una superficie cón due punti doppi; mentre lo spazio tangente in un punto di una retta non speciale non puó mai essere tangente a $V$ in un secondo punto di questa stessa reta. 
al $n .^{\circ} 5$ ). E nella rigata $\Sigma$, formata dalle rette speciali $p$, ogni generatrice dovrà appoggiarsi alla successiva; sicchè questa rigata sarà appunto una sviJuppabile, c. s. v. d. I piani tangenti di questa sviluppabile, i quali devono toccarla lungo le singole rette $p$, saramno gli stessi piani $\pi$ sopra considerati, che sono appunto tangenti a $V$ lungo le medesime rette.

Le $\infty^{1}$ rette speciali contemute in una varietà cubica $V$ formano una rigata sviluppabile; e $i$ piani tangenti a $V$ lungo quelle rette sono pure tangenti a questa sviluppabile. Questa sviluppabile si può anche considerare come l'inviluppo degli $\alpha^{2}$ spaizi bitangenti della varietà $V\left({ }^{*}\right)$.

7. Per determinare l'ordine della rigata sviluppabile $\Sigma$ conviene premettere qualche altra considerazione intorno al sistema $\infty^{2}$ delle rette contenute in una varietà cubica generale (**). E ci proponiamo precisamente di determinare l'ordine e il genere della rigata formata da quelle rette della varietà che si appoggiano $a$ un piano generico

Quanto all'ordine, basterà osservare che questa rigata ha la cubica intersezione del piano $\xi$ colla varietà $V$ come direttrice sestupla, e che ogni spazio $S_{3}$ passante per questo piano la incontra ulteriormente secondo 27 ge-

(*) Si puo dimostrare facilmente anche per assurdo whe la rigata \$ deve essere una sviluppabile. Infutti per ogni rigata non sviluppabile appartenente a uno spazio superiore a $S_{3}$ esiste, corrispondentemente a ogni generatrice $g$ non singolare, un determinato spazio $S_{\mathrm{g}}$ che deve considerarsi come congiungente questa generatrice alla consecutiva fossia $\grave{d}$ posizione limite dello spazio $S_{3}$ che congiunge la generatrice $g$ a unaltra, variabile, che si avvieina indefinitamente alla prima entro la rigata). In questo particolare $S_{3}$ sono contenuti i piani tangenti alla rigata proposta nei singoli punti di $g$, i quali formano un fascio proiettivo alla puntegriata dei loro punti di contatto. Ogni piano di questo fascio è tangente alla rimata in uno e un solo punto di $g$; e ogni altro $S_{3}$ passante per $g$ non potri contenere che uno solo di questi piani, e non potra perció toccare la rigata proposta che in un solo punto della generatrice $g$. Ora, se $p$ è una generatrice qualunque della nostra

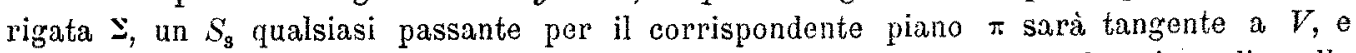
percio anche a $\Sigma$, in due punti, in generale distinti, di quella retta; e al variare di quello spazio $S_{3}$ nel fascio $\pi$ varieranno pure questi due punti di contatto sopra $p$, contrariamente a quanto si è veduto che deve avvenire per ognni generatrice non singolare di una rigata sghemba.

(**) Questo sistema di rette è certo irriducibile: esso potrebbe spezzarsi in due o più altri (distinti) soltanto quando la varieta cubica su cui giace avesse qualche punto doppio. Per maggiori dettagli si veda la mia Nota: Sulle superficie algebriche..., negli Atti della, R. Ace. di Torino, vol. $39 ; 190 \%$. 
neratrici (le 27 rette contenute nella superficie intersezione del medesimo $S_{3}$ colla varietà $V$ ): perciò l'ordine della nostra rigata sarà $=3.6+27=45$.

Per determinarne il genere, osserveremo che su questa stessa rigata, considerata come ente algebrico $\infty^{1}$ di rette, gli spazi $S_{3}$ passanti per il piano $\xi$ segano gruppi di 27 generatrici formanti una serie lineare $g_{57}^{4}$; e in questa serie lineare si hanno gruppi con elementi multipli; cioè due almeno delle 27 rette coincidono, soltanto quando lo spazio $S_{3}$ è tangente alla varietà $V(*)$. Ora di questi spazi tangenti, nel fascio $\zeta$, ve ne sono $24\left(\mathrm{n}^{\circ} 1\right)$; e in ciascuno di essi vi sono 6 rette (quelle che passano per il punto di contatto, ossia per il punto doppio della superficie sezione) che ranno contate due volte. In tutta la serie lineare vi saranno dunque $6.24=144$ elementi doppi; e jl genere incognito $p$ della nostra rigata dovrà perciò soddisfare alla relazione:

$$
2(27+p-1)=144
$$

dalla quale si ricava $p=46$.

Le rette di una varietd cubica generale che si appoggiano a un viano generico formano una rigata di ordine 45 e genere 46.

Questa rigata si può considerare come l'intersezione del sistema $\infty^{2}$ di rette contenuto nella varietà $V$ col complesso lineare singolare formato da tutte le rette dello spazio $S_{4}$ che si appoggiano al piano dato. $\mathrm{E}$, più generalmente, la rigata intersezione del medesimo sistema $\infty^{2}$ di rette con un complesso lineare affatto qualunque avrà anch'essa il medesimo ordine $45 \mathrm{e}$ lo stesso genere 46 . In altri termini, nello spazio lineare $S_{9}$ definito dalle dieci coordinate omogenee di retta $r_{i k} \doteq\left(x_{i} y_{k}\right)(i=k=k ;, k=0,1,2,3,4)$ jl sistema $\infty^{2}$ delle rette contenute nella varietà cubica. $V$ si potrà considerare come una superficie di ordine 45 , a sezioni di genere $46\left(^{* *}\right)$.

In particolare, se il piano dato $\xi$ è tritangente a $V$, ossia incontra questa varietà secondo una terna di rette, la rigata $R^{\text {ts }}$ delle rette contenute in $V$ e incidenti a questo piano si spezzerà in tre rigate di ordine $\frac{45}{3}=15$. E siccome queste rigate hanno a due a due cinque generatrici a comune (delle

(*) Poichè in una stuperficie di $3 .^{\circ}$ ordine priva di punti doppi le 27 rette sono sempre tutte distinte (Kresr, Mem. cit, Math. Ann., VI, p. 560).

(*) I principali caratteri di questa superficie sono stati determinati da me in un altro lavoro (Sul sistema $\infty^{2}$ di rette contenuto in una varieta culica generale dello spasio a quattro climensioni; Atti della R. Acc. di T'orino, vol. 39. 1904 ). 
quali quattro appartengono al punto d'incontro delle loro direttrici rettilinee, e una al piano di queste direttrici), cosi il loro genere $p^{\prime}$ si potrà determinare valendosi della solita formola che esprime il genere di una curva com. posta; in questo caso:

da cui

$$
3 p^{\prime}+3.5-2=46
$$

$$
p^{\prime}=11
$$

Le rette di una varietà cubica generale che si appoggiano a una qualunque fra queste stesse rette formano una rigata di ordine 15 e genere 11.

8. L'ordine della sviluppabile $\Sigma$ formata dalle rette speciali contenute in $V$ sarà dato dal numero delle sue generatrici che si appoggiano a un piano generico, ossia dal numero di quei punti della curva di $3 .^{\circ}$ ordine $\chi^{3}$, intersezione di questo piano colla varietà $V$, pei quali coincidono due delle sei rette di $V$ che ne escono. Ora le $\infty^{1}$ rette di $V$ che si appoggiano a quel piano, e quindi alla curva $\chi^{3}$, formano una rigata di genere 46 ; e in questa rigata le sestuple di rette uscenti dai singoli punti di $\chi^{3}$ formano un'involuzione ellittica $I_{6}^{4}$, della quale si domandano gli elementi doppi. Il numero di questi elementi è dato dalla formola di Zeuthen-Segre $\left({ }^{*}\right)$ :

$$
y=2\left(p^{\prime}-1\right)-2 \mu(p-1)
$$

per $\mu=6, p=1, p^{\prime}=46$; onde $y=90$.

La sviluppabile 乏 è dunque di ordine $90\left(^{* *}\right)$. E vi saranno perciò $\frac{90}{3}=30$ sue generatrici che si appoggiano a una qualsiasi retta non speciale contenuta in $V$. - Nella rigata di ordine 15 e genere 11 formata da tutte

(*) Zeuthen: Nowvelle ciemonstration... (Math, Ann. III, p. 150); SwGRE: Introduzione alla geometria sopra un ente algebrico semplicemente infinito (Ann. di Mat., ser. II, t. 22; n. $\left.{ }^{\circ} 40\right)$.

(**) Il Sig. Enriques nella Mem. cit. (p. 32) trova che questa superficie è di ordine 120. Ma nella corrispondenza $(20,20)$ da lui considerata sulla retta non speciale $r$ sono punti uniti anche le 5 intersezioni di $r$ colla varietà Hessiana, per ciascuna delle quali due delle rimanenti rette di $V$ che ne escono stanno in un medesimo piano per $r$; e ciascuna di queste intersezioni assorbe due coincidenze. Percio sono soltanto $40-5.2=30$ le coincidenze dovute a rette speciali che si apposgiano ad $r$. 
le rette di $V$ che si appoggiano a questa retta non speciale i gruppi di 5 generatrici uscenti dai singoli punti della direttrice rettilinea formano una serie lineare $g_{5}^{1}$, della quale quelle 30 generatrici di $\Sigma$ sono precisamente i $2(5+11-1)==30$ elementi doppi.

9. L'ordine della sviluppabile $\Sigma$ si può determinare anche per un'altra via, la quale permette di trovarne in pari tempo il genere.

Le quadriche polari (rispetto a $V$ ) dei punti di un piano qualsiasi $\xi$ formano una rete, e le rette contenute in tutte queste quadriche formano a lor volta un complesso cubico $\mathrm{r}$, contenente tutte le $\infty^{3}$ rette speciali (n. ${ }^{\circ}$ 2) e perciò anche le $\infty^{1}$ generatrici di $\Sigma$. D'altra parte una retta non speciale contenuta in $V$ appartiene a un solo fascio di quadriche del sistema polare; e queste sono precisamente le quadriche polari dei punti della retta medesima. Perciò le rette contenute in pari tempo nella varietà $V$ e nel complesso $\Gamma$ o saranno generatrici di $\Sigma$, oppure dovranno appoggiarsi al piano $\xi$. E poichè queste ultime formano una rigata di ordine $45\left(n .{ }^{\circ} 7\right)$, così $\Sigma$ sarà di ordine $3.45-45=90$.

La rigata (sviluppabile) $\Sigma$ può dunque segarsi dalla varietà $\infty^{2}$ delle rette contenute in $V$, e in infiniti modi, mediante un complesso cubico passante per l'intersezione della medesima varietà $\alpha^{2}$ con un complesso lineare di rette. Essa appartiene dunque al sistema lineare doppio di quello che sulla stessa varietà $\propto^{2}$ segano i complessi lineari di rette; e siccome quest'ultimo sistema è di ordine 45 e genere 46 , così il primo sarà di genere

$$
2.46+45-1=136 \text {. }
$$

Questo stesso sarà dunque, in generale, il genere della rigata (sviluppabile) $\Sigma$; e lo sarà certo fin tanto che essa non abbia elementi doppi.

Ora una generatrice doppia di $\Sigma$ non potrebbe essere (come si vede immediatamente) che una generatrice di regresso, tale quindi che per ogni suo punto non soltanto due, ma tre delle rette di $V$ che ne escono vengano con essa a coincidere. Allora tutti gli $S_{3}$ bitangenti a $V$ in punti di questa retta segherebbero $V$ secondo superficie con due punti doppi biplanari (perchè in questo caso soltanto la retta che congiunge $\mathrm{i}$ due punti di contatto conta per ciascuno di essi come tre fra le sei che ne escono), e $V$ ammetterebbe lungo quella retta un piano osculatore fisso (ossia un piano che l'incontra secondo tale retta soltanto, contata tre volte). E l'enumerazione delle costanti ci mo- 
stra facilmente che sopra una varietà cubica generale non esistono rette cosi fatte $(*)$.

Concludiamo pertanto: Sopra una varietà cubica generale le $\alpha^{-1}$ rette speciali formano una rigata sviluppabile di ordine 90 e genere 136.

10. Si può determinare facilmente anche l'ordịne della varietà a tre dimensioni formata dagli $\alpha^{1}$ piani tangenti $\pi$ della sviluppabile $\Sigma$. Esso è dato infatti dal numero di quei piani $\pi$ che si appoggiano a una retta generica $s$, ossia dal numero degli spazi bitangenti che passano per questa retta. Ora gli spazi tangenti a $V$ e passanti per la retta $s$ hanno i loro punti di contatto sulla superficie $\varphi^{4}$ base del fascio formato dalle quadriche polari dei punti di $s$ medesima; e perciò quelli fra essi che sono bitangenti a $V$ avranno i punti di contatto nelle intersezioni di questa superficie $f^{4}$ colla sviluppabile $\Sigma$, le quali sono in numero di $4.90=360$. Saranno dunque $\frac{360}{2}=180$ gli spazi bitangenti che passano per $s$; e questo sarà pure l'ordine della varietà a tre dimensioni formata dai piani $\pi$, tangenti alla sviluppabile $\Sigma$.

Questa varietà avrà a comune con $V$ una superficie di ordine $3.180=540$, della quale farà parte la sviluppabile $\Sigma^{90}$ contata due volte, poichè essa ̀̀ doppia per la varietà formata dai piani $\pi$. La parte residua, di ordine 360 , sarà la rigata luogo di quelle rette di $V$ che sono "coniugate" (cfr. n. ${ }^{0} 5$ ) delle rette speciali $p$; e questa nuova rigata non sarà in generale una sviluppabile, perchè una sua generatrice qualunque non potrà essere incidente alla consecutiva, a meno di non essere anch'essa una retta speciale (n. ${ }^{0} 6$ ).

11. Dello spigolo di regresso $\sigma$ della sviluppabile $\Sigma$ si conoscono ora tre caratteri: il genere 136 ; il primo rango, ossia l'ordine $=90$ della rigata sviluppabile formata dalle sue tangenti; e il secondo rango, ossia l'ordine $=180$

(*) Infatti l'imporre a una variota cubica di incontrare un dato piano secondo una retta assegnata, contata tre volte, equivale a 9 condizioni lineari (come se si trattasse di una data cubica piana qualsiasi); e perciò una varietà soddisfacente a queste condizioni dipende ancora da $34-9=25$ parametri. Daltra parte la scelta di quel piano, in $S_{4}$, dipende da 6 costanti, e la scelta della retta nel piano da altre 2 costanti; sicchè, complessivamente, una varieti con piano osculatore fisso lungo una retta dipendera soltanto da $6+2+25=33$ parametri, mentre le varietai culjohe di $S_{4}$ sono in numero di $\infty^{34}$. 
della varietà formata dai suoi piani osculatori, cioè dai piani tangenti di questa sviluppabile.

Indicando pertanto con $m$ l'ordine della linea $\sigma$, e $\operatorname{con} \beta$ il numero delle sue cuspidi, avremo le due relazioni (*):

$$
\begin{aligned}
& 2(m+136-1)-\beta=90 \\
& 3(m+2.136-2)-2 \beta=180
\end{aligned}
$$

dalle quali si ricava $m=270, \beta=720$ (e questi valori risulteranno anche confermati in seguito per altre vie; cfr. n. 12 e 19).

Lo spigolo di regresso $\sigma^{270}$ si potrà considerare come il luogo delle intersezioni delle coppie di rette speciali $p$ consecutive, e sarà perciò il luogo di quei punti di $V$ pei quali non soltanto due, ma tre delle sei rette che ne escono sono venute a coincidere colla corrispondente $p$ (ivi tangente a $\sigma$ ). E per ognuna delle 720 cuspidi di $\sigma$ coincideranno colla $p$, che sarà la tangente cuspidale, quattro delle medesimo sei rette. D'altra parte una superficie cubica dello spazio ordinario la quare abbia un punto doppio tale che le 6 rette di essa uscenti da questo punto non siano tutte distinte ha anche un secondo punto doppio (che può essere infinitamente vicino al primo) sopra ognuna di queste rette la quale conti come due almeno fra le 6 , e anzi, quando vi è una retta che conta come tre o quattro fra le 6, questo secondo punto doppio è un punto biplanare, rispett. del tipo $B_{3} \circ B_{4}$ di Schlaefli $\left(^{*}\right.$ ) (ossia che produce un abbassamento rispett. di tre o quattro unità nella classe della superficie). Concludiamo pertanto:

Lo spazio $S_{3}$ tangente a $Y$ in un punto della curva $\sigma^{270^{\circ}}$ tocca questa varietì anche in un secondo punto, appartenente alla medesima retta speciale $\mathrm{p}$, e tale che la superficie sua intersezione con $V$ ha in questo secondo

(*) Segre: Mem, cit. Introduzione alla geometria..., n. $42-43$. La linea $\sigma$ non avrà, in generale, alcun flesso; perchè la tangente ad essa in un tale punto sarebbe generatrice di regresso della sviluppabile $\Sigma$, e di queste generatrici abbiamo veduto che in generale non ve ne sono $\left(n .^{\circ} 9\right)$.

$(* *)$ On the distrilution of surfaces of the third order into Species... (Phil. Trans. 1863, p. 193 e seg.). Le proprietà sopra enunciate si deducono tutte facilmente dall' esame dei diversi casi di superficie con punti doppi considerati nella Memoria di Schlafful, oppure ánche dalla rappresentazione piana di queste superficie ottenuta mediante proiezione dal primo punto doppio. 
punto un punto doppio biplanare. Più particolarmente, se il punto considerato sulla curva $\sigma$ è una delle sue $\gamma 20$ cuspidi, questo punto doppio biplanare sarà del tipo $B_{4}$, ciò̀ di quelli che equivalgono a una coppia di punti doppi conici infinitamente vicini (sicchè lo spazio $S_{3}$ dovrà allora considerarsi come un particolare spazio tritangente, con due punti di contatto infinitamente vicini; efr. n. ${ }^{0} 18$ ).

Lo spazio $S_{3}$ tangente a $V$ in un punto della curva $\sigma^{270}$ coincide altresì collo spazio osculatore a questa curva in quel medesimo punto, perchè entrambi possono considerarsi come determinati da una coppia di piani tangenti $\pi$ consecutivi della sviluppabile $\Sigma$. Tra questi $S_{3}$, quelli che passano per un punto dato qualsiasi avranno i loro punti di contatto nelle intersezioni della linea $\sigma$ colla quadrica polare di questo punto, le quali sono in numero di $2.270=540$. La curva c è dunque di classe $n=540$; valore che è confermato dalla formola:

$$
n=4(m+3 p-3)-3 \beta
$$

dove, come già sappiamo, $m=270 ; p=136 ; \beta=720$.

Gli spazi $S_{3}$ jperosculatori alla curva $\sigma^{279}$ saranno elementi doppi (di regresso) della varietà $\infty^{1}$ formata dagli $S_{3}$ osculatori a questa stessa curva. Ora, perchè lo spazio $S_{3}$ osculatore $a \sigma$ in un punto $P$ conti rispetto a ogni suo punto come due fra i 540 spazi osculatori che ne escono, è necessario e sufficiente che per le quadriche polari di tutti i suoi punti due (almeno). delle 540 intersezioni con $\sigma$ coincidano in $P$. Ciò avviene certamente per ognuna delle 720 cuspidi di $\sigma$; sicchè lo spazio $S_{3}$ osculatore a $\sigma$ in ognuna di queste cuspidi sarà un elemento stazionario della varietà $\infty^{1}$ degli spazi osculatori medesimi (e il carattere comune di questi punti singolari sarà espresso dai numeri $\left.i=2, i_{1}=3, i_{2}=4, i_{3}=6(*)\right)$. All'infuori di questo caso, la condizione sopra accennata non sarà verificata se non quando quelle $\infty^{3}$ quadriche risultino tutte tangenti alla curva $\sigma^{z 70}$ nel punto $P$, e abbiano perciò in questo punto una tangente fissa; sicchè questo punto dovrà appartenere alla varietà Hessiana (n. ${ }^{0} 3$ ). Il numero o di questi punti semplici, nei quali la linea $\sigma$ ammette uno spazio iperosculatore, sarà dato dalla relazione:

$$
\alpha=5(m+4 p-4)-5 \beta=5(270+4.135-720)=450
$$

(*) Segre, Mem, cit, n, 43 . 
e vedremo in seguito (n." 13) che queste sono anche le sole intersezioni (distinte) della linea - colla varietà Hessiana.

12. Proponiamoci ora di determinare come sia composta la curva intersezione della sviluppabile $\Sigma^{90}$ colla varietà Hessiana.

Ricordiamo perciò che ogni generatrice $p$ della sviluppabile $\Sigma^{00}$ è tangente alla Hessiana nei due punti doppi $M, N$ dell'involuzione segata su di essa dalle quadriche del sistema polare $\left(n^{0} 4\right)$, e incontra perciò questa varietà in un solo punto ulteriore $R\left(^{*}\right)$. L'intersezione di $\Sigma^{90}$ colla varietà Hessiana si comporrà dunque di due parti distinte: una linea di contatto $\mu$, luogo dei punti $M$ e $N$ delle singole generatrici; e una linea $\rho$ di semplice intersezione, luogo dei punti $R\left({ }^{* *}\right)$.

L'ordine della linea : si può determinare per mezzo della considerazione seguente $\left(^{* *}\right)$. Sia $l^{00}$ una sezione ipcrpiana generica della rigata $2^{00}$. Ogni punto di essa avrà sulla medesima generatrice $p$ che lo contiene uno e un solo punto suo coniugato nell'involuzione $I_{16}$; e la linea $l$ luogo di questi nuovi punti sarà anch'essa di ordine 90 , poichè le due linee $l$ e $l$, coniugate fra loro punto per punto nella $I_{: b}$, dovranno essere incontrate da ciascuna quadrica del sistema polare in un medesimo numero di punti, e avranno perciò lo stesso ordine. Segue da ciò che queste due linee arranno 90 punti a comune - le intersezioni di $l^{\prime}$ collo spazio $S_{3}$ contenente la linea $l$-; e questi 90 punti, che saranno punti $M \circ N$ di altrettante generatrici $p$, saranno tutte e sole le intersezioni della curva $\mu$ collo spazio $S_{3}$ sopra accennato. La curva $\mu$ è dunque di ordine 90.

Ora l'intersezione complessiva della superficie $\Sigma^{90}$ colla varietà Hessiana deve essere di ordine $90.5=450$; e la linea $\mu^{90}$, essendo loro linea di contatto, va contata due volte come parte di questa intersezione. L'intersezione residua $\rho$ sarà perciò di ordine $450-2.90=270$.

La sviluppabile $\Sigma^{90}$ e la varietà Hessiana si toccano lungo una linea di

(*) Questi tre punti $M, N, R$ sono anche le intersezioni della stessa retta $p$ colla sua $C_{3}^{7}$ residua (cfr. n. ${ }^{i} 3-4$ ).

(*) Di questa intersezione non fa parte, in generale, nessuna generatrice $p$ : poichè, se ciò avvenisse, tutti gli $S_{3}$ bitangenti a $V$ in coppie di punti di questa retta incontrerebbero $V$ secondo superficie con due punti doppi biplanari, e vi sarebbe per conseguenza lungo la stessa retta un piano osculatore fisso; il che, in generale, non avviene (n. ${ }^{\circ} 9$ ).

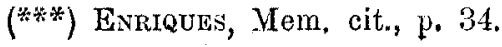


ordine 90 (luogo dei punti doppi delle involuzioni che le quadriche del sistema polare segano sopra le rette $p$ ), e si incontrano ulteriormente secondo una linea di ordine $2 \%$.

Gli spazi $S_{3}$ tangenti a $V$ in punti $(M \circ N)$ della linea $\mu^{90}$ si potranno considerare come spazi bitangenti coi due punti di contatto infinitamente vicini sopra la corrispondente retta $p$; essi segheranno $V$ secondo superficie con un punto doppio biplanare del tipo $B_{4}$, i cui due piani tangenti passeranno entrambi per la retta $p$. Invece lo spazio tangente in un punto $R$ della linea $\rho$ toccherà $V$ in un secondo punto $P$, in generale distinto da $R$, che sarà precisamente il coniugato armonico di $R$ rispetto ai due punti $M, N$ della medesima generatrice $p$; e segherà $V$ secondo una superficie che ha in $R$ un punto doppio biplanare (in generale del tipo $B_{3}$ ) e in $P$ un punto doppio conico. Percio tre delle 6 rette di $V$ che escono da questo purito $P$ dovranno coincidere colla $p \equiv P R$, ossia il punto $P$ starà sullo spigolo di regresso $\sigma^{270}\left(\mathrm{n} .0^{0} 11\right)$. Concludiamo pertanto:

Gli spazi $S_{3}$ tangenti a $V$ nei singoli punti di una delle due linee $\rho^{9 \pi 0}$


linee (e sulla medesima retta $p$ ). Queste due linee sono coniugate nell' involuzione $I_{10}$; ogni punto dell' una ha uno e un solo coniugato sopra l'altra, e in questi due punti coniugati la varietà $V$ ha sempre un medesimo $S_{3}$ tangente. Lo stesso ragionamento applicato poc'anzi alle due linee $l$ e $l$ permetterebbe di concludere che anche $p$ e $\sigma$ devono avere il medesimo ordine; e si ha in cio una conferma dei risultati precedenti.

Le proprietà note di una superficie cubica con un punto doppio conico e un punto biplanare permettono altresi di affermare che la retta coniugata di una retta speciale $p\left(n .^{\circ} 5\right.$ ) si appoggia a quest'ultima nel suo punto $R$ (ossia in quel punto che è intersezione semplice di $p$ colla Hessiana).

13. Ogni punto $K$ comune alla linea $\sigma^{: 70}$ e alla varietà Hessiana di $V$ dovrà appartenere alla curva intersezione complessiva della sviluppabile $\Sigma^{20}$ con quest'ultima varietà, e perciò a una almeno delle due linee $\mu^{90}$ e $\rho^{270}$; anzi a entrambe queste linee, perchè sopra la retta $p$ che contiene quel punto (facendo uso delle solite notazioni) la quaderna di punti $M N R K$ deve essere un gruppo armonico, e perciò $K$ non può coincidere nè con $R$, nè con uno dei punti $M \circ N$, senza che avvengano l'una e l'altra cosa in pari tempo. Lo spazio $S_{3}$ tangente a $V$ in uno di quei punti $K$ incontrerà $V$ secondo una superficie con un punto doppio biplanare del tipo $B_{5}$ (che può considerarsi 
come proveniente dall'avvicinarsi indefinitamente di un punto doppio conico e di un punto biplanare del tipo $B_{3}$ ).

Ora le intersezioni della curva $\sigma^{270}$ colla varietà Hessiana sono complessivamente in numero di $270.5=1350$. In ognuna di queste intersezioni la retta $p$ tangente a $\sigma^{z z 0}$ è tangente tripunta della varietà Hessiana (perchè sono venute a coincidere su di essa l'intersezione $R$ e uno dei punti di contatto $M, N)$; e il piano $\pi$ osculatore a $\sigma$ è anch'esso tangente alla Hessiana, perchè contiene, oltre alla $p$, le tangenti nel medesimo punto alle linee $\mu$ e $\rho$ contenute nella Hessiana (le quali tangenti sono certo distinte da $p$ ). In ciascuno di questi punti la linea $\sigma^{2 \% 0}$ e la varietà Hessiana avranno perciò un contatto di $2 .^{\circ}$ ordine $\left(^{*}\right)$; e il numero delle loro intersezioni distinte sard soltanto $\frac{1350}{3}=450$, ossia non vi saranno altre intersezioni all'infuori di quelle già considerate al $n .{ }^{\circ} 11$.

Possiamo dunque dire, riassumendo: La curva oro ha a comune colla varietà Hessiana 450 punti (in generale distinti), in ciascuno dei quali essa

(") Nello spazio $S_{3}$ una linea e una superficie hanno in un punto, semplice per entrambe, un contatto che è certo di $2_{*}^{\circ}$ ordine (almeno) quando la tangente alla linea è tangente principale della superficie e il piano osculatore alla linea coincide col piano tangente della superficie - quando cioè, in altri termini, la linea si comporta nelle vicinanze di quel punto come un'asintotica della superficiè -; avvertendo inoltre che questa condizione è bensì sufficiente perchè vi sia un contatto di $20^{\circ}$ ordine, ma non è certo necessaria.

Infatti, in coordinate carteșiane, una superficie passante per l'origine delle coordinate, tangente in questo punto al piano $z=0$, e avente per tangente principale la retta $y=z=0$, si può rappresentare, nelle vicinanze dell'origine stessa, con un'equazione del tipo:

$$
z=a_{1} x y+a_{2} y^{2}+a_{3} x z+a_{1} y z+a_{5} z^{2}+\ldots
$$

dove i termini non seritti sono di grado superiore al secondo. E una linea passante (semplicemente) per l'origine, ivi tangente alla retta $y=z=0$ e osculatrice al piano $z=0$, si può rappresentare parametricamente, nelle vicinanże dello stesso punto, colle equazioni:

$$
x=\alpha \rho+\alpha_{1} \rho^{2}+\ldots \quad y=\beta_{1} \rho^{2}+\ldots \quad z=\gamma_{2} \rho^{3}+\ldots
$$

Sostituendo ora queste espressioni nell'equazione precedente, si ottengono soltanto termini contenenti $\rho$ ad esponente $\supseteq 3$; sicchè l'origine assorbirc tre almeno delle intersezioni di questa linea colla superficie proposta, c. s. v. d.

Similmente si proverebbe che, nello spazio $S_{4}$, una linea e una varieta $M_{3}$ hanno in un punto, semplice per entrambe, un contatto almeno di $2 .^{\circ}$ ordine ogni qual volta la tangente alla linea è tangente tripunta della $M_{3}$ e in pari tempo il sio piano osculatore $\dot{e}$ tangente a questa $M_{3}$. 
è osculatrice a quest'ultima varietà. Gli spazi $S_{3}$ tangenti a $V$ in questi punti sono iperosculatori $a \sigma, e$ incontrano $V$ secondo superficie con munto doppio biplanare del tipo $B_{5}$.

\section{Spazi tritangentt.}

14. La sviluppabile $\Sigma^{90}$, già considerata ai n. 6 e seg., ha una curra doppia $\%$ luogo delle intersezioni di coppie di generatrici $p$ (non consecutive).

Le rette della varietà $V$ che si appoggiano a una retta speciale $p$ formano anch'esse (n. ${ }^{0} 7$ ) una rigata di ordine 15 e genere 11 ; però da ogni punto della direttrice rettilinea considerata escono soltanto 4 generatrici variabili di questa rigata, formanti $\mathrm{j}$ gruppi di una serie lineare $g_{4}^{4}$. Questa serie lineare avrà dunque $2(4+11-1)=28$ elementi doppi, che saranno costituiti da altrettante rette speciali $p$. Vale a dire: Ogni retta speciale $p$ contenuta nella varietì $V$ si appoggia a 28 altre rette consimili; ovverosia: La sviluppabile $\Sigma^{90}$ ha (all'infuori dello spigolo di regresso) una curva doppia che incontra ogni sua generatrice in 28 punti.

Ora, quando da un punto $X$ della varietà $V$ escono due diverse rette speciali $p, p^{\prime}$, lo spazio $S_{3}$ tangente a $V$ in quel punto dovrà toccare questa varietà anche in un secondo punto di ciascuna di queste due rette; esso sarà dunque uno spazio tritangente, e la congiungente di questi due ulteriori punti di contatto (in generale distinti da $X$ ) sarà una terza retta speciale $p^{\prime \prime}$, contenuta nel piano $p p^{\prime}$. Perciò le 28 rette speciali incidenti a $p$ dovranno distribuirsi a coppie in 14 piani passanti per $p$.

Nel fascio formato dagli spazi bitangenti a $V$ in coppie di punti di una retta speciale p vi sono 14 spazi tritangenti, ciascuno dei quali tocca la varietà $V$ anche in un terzo punto, non appartenente in generale a quella retta. Questi tre punti di contatto apparterranno sempre alla curva doppia $\gamma$ della sviluppabile $\Sigma^{n}$.

15. A quest'ultimo risultato si può arrivare anche per mezzo di altre considerazioni $\left(^{*}\right)$, le quali ci permetteranno pure di determinare l'ordine della curva $\gamma$.

$\left(^{*}\right)$ Exriques, Mem. cit, p. 33. 
Sia $p$ una retta speciale contenuta nella varietà $V$, e $\pi$ il piano tangente fisso lungo di essa comune a questa varietà e alla sviluppabile $\Sigma^{90}$. Ogni spazio $S_{3}$ del fascio $\pi$ sarà bitangente a $V$ in punti della retta $p$; e avrà due dej propri poli in questi suoi punti di contatto, e i rimanenti 14 sulla curva $C_{3}^{7}$ " residua" di $p\left(n .^{0} 2\right)$. Questo spazio sarà dunque tritangente a $V$ ogni qual volta uno dei 14 suoi poli ulteriori apparterrà a $V$ stessa - senza però cadere, in generale, sopra $p-$; e il numero degli spazi tritangenti contenuti nel fascio $\pi$ sarà dato perciò dalle intersezioni della varietà $V$ colla curva $C_{3}^{7}$ sopra accennata, escluse quelle che appartengono alla retta $p$.

Ora la retta $p$ è una (anzi l'unica) trisecante di questa curva $C_{3}^{7}$, e l'incontra precisamente nei tre punti, in generale distinti, che essa $p$ ha a comune colla varietà Hessiana, e che al $n .^{\circ} 12$ abbiamo indicati colle lettere $M, N, R$. Ciascuno di questi sarà un punto comune alla $C_{3}^{7}$ e alla varietà $V$; e anzi in ciascuno di essi la $C_{3}^{7}$ è certo tangente a $V$. Infatti la tangente a $C_{3}^{7}$ in uno qualunque di questi punti è pure tangente, nel medesimo punto, alla quadrica polare di tale punto (la quale passa per $C_{3}^{7}$ ), e percio anche alla varietà $V$ (la quale è ivi tangente a questa quadrica). Dico ancora, di più, che nel punto $R$ la $C_{3}^{7}$ ha con $V$ un contatto di $20^{\circ}$ ordine. Infatti il piano $\pi$ incontra la varjetà $V$ secondo una cubica composta della retta $p$ contata due volte e di una retta ulteriore passante per $R\left(\right.$ n. $\left.^{0} 12\right)$ : perciò le quadriche polari dei punti di $\pi$ incontreranno questo stesso piano secondo coniche composte della retta $p$ come parte fissa e di una retta ulteriore, variabile, passante anche per $R$; esse saranno dunque tutte tangenti al piano $\pi$ nel punto $R$, e non vi avranno fuori di questo piano altri elementi tangenti a 'comune (*). Di qui si trae che la tangente in $R$ alla linea $C_{3}^{z}$, la quale insieme alla retta $p$ è curva base di quella rete di quadriche, dovrà stare nel pjano $\pi$, e sarà perciò una tangente tripunta della varietà $V$. Per assicurarsi dunque che la $C_{3}^{7}$ abbia in $R$ un contatto di $20^{\circ}$ ordine con $V$, basterà far vedere (cfr. n. ${ }^{0} 13$ ) che il suo piano osculatore in $R$ è contenuto nello spazio $S_{3}$ ivi tangente a $V$; e ciò sarà verificato analiticamente al n. ${ }^{\circ}$ seg.

Pertanto, delle $3.7=21$ intersezioni della nostra $C_{3}^{7}$ colla varietà $V$, tre cadranno nel punto $R$ e due in cinscuno dei punti $M, N$; ne rimangono dunque, fuori di questi punti, ossia fuori di $p, 21-(3+2.2)=14$; c.s. $\mathbf{\text { v. d. }}$

${ }^{*}$ ) Se no esse arrebbero tutte in $R$ il medesimo $S_{3}$ tangente; ed è facile verificare che eiò non avviene. 
16. Dalla Mem. cit. di Schla ferd (p. 216-17) risulta che all'equazione di una superficie cubica dello spazio ordinario con un punto doppio biplanare (del tipo generale $B_{3}$ ) e un punto doppio conico si può dare la forma:

$$
x_{1} x_{3} x_{4}+x_{2}^{2} x_{3}+\left(a x_{1}+x_{2}\right)\left(b x_{1}+x_{2}\right)\left(c x_{1}+x_{2}\right)=0
$$

dove $a, b, c$ sono certe costanti, e $\mathrm{i}$ due punti doppi cadono rispett. nei due punti fondamentali [4] e [3].

Segue da ciò che una varietà cubica dello spazio $S_{4}$, la quale dallo spazio $x_{0}=0$ sia incontrata secondo una superficie così fatta, si potrà rappresentare coll' equazione :

$$
x_{0} t+x_{1} x_{3} x_{4}+x_{2}^{9} x_{3}+\varphi=0
$$

dove $f \equiv \sum_{0}^{4} a_{i k} x_{i} x_{k}$ è una forma quadratica, coi coeffirienti $a_{33}$ e $a_{44}$ diversi da zero se la varietà non ha punti doppi; e $\varphi$ indica per brevità il prodotto $\left(a x_{1}+x_{2}\right)\left(b x_{1}+c_{2}\right)\left(c x_{1}+x_{2}\right)$. Il piano $x_{0}=x_{1}=0$ è tangente alla varietà lungo l'intera retta speciale $x_{0}=x_{1}=x_{2}=0$, e la incontra ulteriormente secondo la retta $x_{0}=x_{1}=x_{2}+x_{3}=0$.

Designando con indici le derivazioni rispetto alle diverse variabili, si vede che le quadriche polari dei punti del piano $x_{0}=x_{1}=0$ :

$x_{2}^{\prime}\left(x_{0} f_{2}+2 x_{2} x_{3}+\varphi_{2}\right)+x_{3}^{\prime}\left(x_{0} f_{3}+x_{1} x_{1}+x_{2}^{2}\right)+x_{1}^{\prime}\left(x_{0} f_{1}+x_{1} x_{3}\right)=0$

sono tutte tangenti nel punto fondamentale [4] a questo medesimo piano; e formano una rete, la cui curva base si compone della retta $x_{0}=x_{1}=x_{2}=0$ e della sua $C_{3}^{7}$ residua. Ogni spazio $S_{3}$ passante per il piano $x_{0}=x_{1}=0$ incontrerà questa $C_{3}^{7}$ nel punto $[4] \equiv R$ da contarsi due volte almeno (perchè la $C_{3}^{\gamma}$ è tangente a quel piano), nei punti $M, N\left(n .^{\circ} 12\right)$ della stessa retta speciale $x_{0}=x_{1}=x_{2}=0$, e in generale in altri tre punti. Ma uno di questi ultimi punti verrà a coincidere con [4] ogni qual volta lo spazio suddetto conterrà il piano osculatore alla $C_{3}^{7}$ in [4] stesso: dico che ciò avviene precisamente per lo spazio $x_{0}=0$ tangente in [4] alla varietà proposta.

Infatti la rete di quadriche (1) viene incontrata dallo spazio $x_{0}=0$ secondo la rete determinata dalle tre quadriche:

$$
2 x_{2} x_{3}+\varphi_{2}=0 ; \quad x_{1} x_{4}+x_{2}^{2}=0 ; \quad x_{1} x_{3}=0
$$

delle quali le prime due sono coni colla generatrice $x_{1}=x_{2}=0$ a comune, ma piani tangenti diversi lungo questa generatrice: essi si incontrano dunque ulteriormente secondo una cubica passante per i loro vertici e che nel ver- 
tice [4] del primo cono è tangente al piano $x_{1}=0$. Perciò dei due piani $x_{1}=0$ e $x_{3}=0$ che insieme costituiscono la terza delle quadriche (2) il primo non arrà a comune colla cubica nessun punto fuori della retta $x_{1}=x_{2}=0$, e il secondo ne avrà soltanto due. Dunque lo spazio $x_{0}=0$ incontrerà la $C_{3}^{\gamma}$ sopra considerata, fuori della retta $x_{0}=x_{1}=x_{2}=0$, soltanto in questi ultimi due punti; dal che segue la verità di quanto avevamo affermato.

17. Al variare della retta speciale $p$ nella sviluppabile $\Sigma^{90}$ di cui essa è generatrice, la sua $C_{3}^{7}$ residua descriverà una certa superficie $F$, luogo di tutti quei poli degli $\infty^{2}$ spazi bitangenti di $V$ che cadono fuori dei rispettivi punti di contatto. Per determinare l'ordine $x$ di questa superficie, basterà osservare che la sviluppabile $\Sigma^{90}$ ha a comune con due quadriche generiche del sistema polare 2.2 .90 punti, $\mathrm{i}$ quali si ripartiscono in 2.90 coppie di punti coniugati nella $I_{16}$; e che le stesse due quadriche dovranno avere a comune colla superficie $F^{x}$ un egual numero 2.90 di gruppi di 14 punti, costituenti insieme con quelle coppie altrettanti gruppi completi dell'involuzione $I_{16}$. Dovrà dunque essere $4 x=2.90 .14$, ossia $x=7.90=630$.

L'intersezione di questa superficie $F^{7.00}$ colla varietà $V$ conterrà come parti le curve $\mu^{90}$ e $\rho^{270}$ considerate al $n^{0}{ }^{12}$, luoghi rispett. dei punti $M$ e $N$ e dei punti $R$ delle singole rette $p$ (i quali appartengono alle $C_{3}^{7}$ residue di queste $p$, e perciò anche alla superficie $F$ ). L'intersezione residua di $F$ e $V$ sarà la curva luogo di quei poli ulteriori degli spazi bitangenti che appartengono ancora a $V$, ossia luogo dei punti di contatto di tutti gli spazi tritangenti a $V$. Quest'ultima curva coinciderà perciò colla curva doppia y della sviluppabile $2^{90}$, già considerata al $\mathrm{n}^{\circ} 14$.

Consideriamo pertanto la curva $C_{3}^{\gamma}$ residua di una $p$ generica, e facciamola variare insieme con questa $p$ descrivendo l'intera superficie $F^{\gamma \cdot 99}$. I punti $M$ e $N$ di quella retta $p$, che sono comuni alla $C_{3}^{\gamma}$ e alla varietà $V$, descriveranno simultaneamente la curva $\mu^{90}$; e siccome in tutti questi punti la curva $C_{3}^{\gamma}$ risulta tangente a $V$ (e non alla $\mu\left(^{*}\right)$ ), così Ja superficie $F^{7.90}$ sarà anch'essa tangente a $V$ lungo l'intera curva $\mu^{90}$. Similmente il punto $R$, altra intersezione della linea $C_{3}^{7}$ colla retta $p$ e colla varietà $V$, descriverà la curva $f^{270}$; e poichè la $C_{3}^{7}$ è sempre osculatrice a $V$ in questo punto $R$

(*) Infatti la tangente alla curva $\mu$, che è contenuta nella sviluppabile $\Sigma^{90}$, deve stare nel piano $\pi$ tangente a questa sviluppabile; mentre invece le tangenti alla $C_{3}$ nei punti $M$ e $N$ non stanno in questo piano. 
(n. ${ }^{i}$ 15-16) senza essere ivi tangente alla $\rho(*)$, così la superficie $F$ generata dalla $C_{3}^{7}$ risulterà osculatrice a $V$ lungo l'intera linea $\rho^{2 ; 0}$. Dall'intersezione complessiva della superficie $F^{r .90}$ colla varietà $V$, la quale è di ordine 3.7 .90 , si staccano dunque la curva $\mu^{30}$ contata due volte e la curva $\rho^{270}$ contata tre volte; perciò la parte residua $\gamma$ sarà di ordine:

$$
3.7 .90-2.90-3.270=10.90=900 \text {. }
$$

La sviluppabile $\Sigma^{90}$ ha una curva doppia $\gamma$ di ordine 900, che è il luogo dei punti di contatto degli spazi tritangenti della varietè $V$ (**). Ognuno di questi spazi tritangenti ha $\mathrm{i}$ suoj tre punti di contatto sulla curva $\gamma^{900}$, e le tre rette che uniscono questi punti a due a due sono rette speciali $p$. Ogni punto semplice della curva $\gamma$ è vertice di uno e un solo di questi " triangoli di contatto "; invece ogni retta $p$ è lato di 14 fra questi triangoli (n. $\left.{ }^{0} 14\right)$.

La tangente alla linea $\gamma^{900}$ in un suo punto qualunque sari l'intersezione dei due piani $\pi$ tangenti alla sviluppabile $2^{90}$ (e quindi anche a $V$ ) lungo le due rette $p$ uscenti da quel punto.

(*) Poichè la linea $\rho$ è intersezione (parziale) della sviluppabile $\Sigma^{90}$ colla varietà Hessiana, la sua tangente in un punto qualunque sarà l'intersezione del piano e dello spazio $S_{3}$ ivi tangenti rispettivamente a queste due varieta. Nel sistema di coordinate di cui abbiamo gia fatto uso al n. ${ }^{\circ} 16$, la tangente alla linea $p$ nel punto $R \equiv[4]$ è rappresentata dalle equazioni $x_{0}=x_{1}=3 x_{2}+x_{3}=0$; mentre la tangente nel medesimo punto alla $C_{3}^{7}$ residua della retta $x_{0}=x_{1}=x_{2}=0$ ha per equazioni $x_{0}=x_{1}=3 x_{2}+2 x_{3}=0$.

(*) L'ordine $=900$ di questa curva $\gamma$ risulta confermato dall'osservazione seguente. Una sezione iperpiana generica della sviluppabile $\mathbf{2}^{90}$ sarà una curva dello spazio $S_{3}$ di ordine 90 e di genere 136 , con 270 cuspidi (nelle intersezioni dello spazio segante collo spigolo di regresso $\sigma^{\mathbf{2 7 0}}$ ) e un punto doppio in ognuna delle intersezioni del medesimo spazio colla curva, $\gamma$. D'altra parte la sviluppabile $2^{90}$, come superficie contenuta in una varietà cubica priva di punti doppi, deve essere l'intersezione completa di questa varietà con un'altra varietà, di ordine 30 (cfr. la mia Nota: Sulle superficie algebriche contenute in una varieta cubica dello spazio a quattro dimensioni; Atti Acc. di Torino, vol. 39, 1904); e perciò ogni sua sezione iperpiana sarà intersezione completa di due superficie rispettivamente di $3^{\circ}$ e di $30^{\circ}$ ordine dello spazio $S_{3}$. Ora la curva intersezione generale di una superficie cubica con una superficie di ordine $n$ è di genere $3\left(\begin{array}{l}n \\ 2\end{array}\right)+1$, e ogni punto doppio o cuspide ne abbassa il genere di un'unità. Indicando dunque con d l'ordine della curva $\gamma$, dovrà essere:

$$
136=3\left(\begin{array}{c}
30 \\
8
\end{array}\right)+1-d-270
$$

e di qui si ricava appunto $d=900$. Quest'osservazione toglie anche ogni dubbio sulla possibilità che le varie intersezioni e i contatti considerati al n. prec. possano eventualmente avere multiplicità superiori a quelle indicate. 
Per un punto gererico di $S_{4}$ passano 600 spazi tritangenti di una data varietà cubica (ossia la sviluppabile formata dagli spazi tritangenti è di classe 600 ). Infatti $i$ punti di contatto di questi spazi tritangenti saranno tulte e sole le intersezioni della curva $\gamma^{900}$ colla quadrica polare del punto considerato; e perciò il inumero di tali spazi sarà $=\frac{2.900}{3}=600$.

18. Nel sistema $\infty^{1}$ degli spazi tritangenti della varietà $V$ vi sono alcuni spazi particolari pei quali sono infinitamente vicini due dei tre punti di contatto, oppure anche (in un certo senso) tutti tre questi punti.

Una superficie cubica di $S_{3}$ con tre punti doppi conici, nella quale due di questi punti si facciano avvicinare indefinitamente, acquista al limite un punto doppio biplanare del tipo $B_{4}$ - oltre al terzo punto doppio conico che rimane - Siccome allora la retta che congiunge questi due punti doppi distinti conta come quattro fra quelle che escono dal punto conico, così quando per una sezione iperpiana della varietà $V$ si presenti questo caso - il punto doppio conico dovrà essere una delle 720 cuspidi della curva $\sigma^{270}$; come anche viceversa (e lo si è già osservato; cfr. n. ${ }^{\circ} 11$ ) lo spazio tangente a $V$ in uno qualunque di questi 720 punti incontrerà $V$ stessa secondo una superficie con un punto doppio conico e un punto biplanare del tipo $B_{\mathbf{4}}$, e dovrà perciò considerarsi come uno spazio tritangente del quale due punti di contatto siano infinitamente vicini. La retta che congjunge i due punti doppi distinti della superficie intersezione di questo spazio con $V$, e la retta asse del punto doppio biplanare (cioè intersezione dei due piani tangenti alla medesima superficie in questo punto) saranno entrambe rette speciali $p, p^{\prime}$; il piano $p p^{\prime}$ di queste due rette sarà tangente alla varietà $V$ e alla superficie sezione lungo l'intera retta $p$; i due punti doppi della superficie sezione apparterranno entrambi alla curva $\gamma^{900}$, la quale anzi nel punto $p p^{\prime}$ sarà tangente alla retta $p^{\prime}$; e la retta $p$ conterà come due fra le 28 rette speciali che si appoggiano a $p^{\prime}\left(^{*}\right)$.

Abbiamo anche già osservato $\left(\mathrm{n}^{\circ} 1\right)$ che sulla varietà $V$ vi sono 60 punti - le intersezioni colla curva doppia della varietà Hessiana - i cui $S_{3}$ tangenti incontrano $V$ secondo superficie con punto doppio uniplanare. Le sei rette di $V$ che escono da un punto siffatto. $X$ coincidono a due a due, e si riducono perciò a tre sole distinte, contenute in un medesimo piano; e lungo

(*) Il punto $p p^{\prime}$ è allora un punto $M \circ N\left(n^{\circ} 12\right)$ per la retta $p^{\prime}$, e un punto $R$ per la retta $p$. Esso appartiene quindi a entrambe le linee $\mu^{90}$ e $p^{270}$; ma appartiene alla p. come punto di $\rho^{\prime}$, e alla $\rho$ come punto di $p$, 
ciascuna di esse la varietà $V$ ammette un piano tangente fisso. Esse sono dunque tutte tre rette speciali, generatrici della sviluppabile $\Sigma^{90}$, e formano un particolare trilatero (degenere) nel quale $\mathrm{i}$ tre lati concorrono in un medesimo punto; ciascuna di esse incontra, fuori di questo punto, soltanto 26 rette speciali, e appartiene perciò soltanto a 13 altri trilateri. Il punto $X$ è triplo per la sviluppabile $\Sigma^{90}$ e per la sua curva doppia $\gamma^{900}$; e sopra ognuna delle tre rette speciali che ne escono esso è uno dei punti doppi $(M, N)$ dell'involuzione segatavi dalle quadriche del sistema polare. Lo spazio tangente a $V$ in ognuno di questi 60 punti fa anche parte del sistema $\infty^{1}$ degli spazi tritangenti, e si può considerare come avente con $T$ tre punti di contatto sovrapposti e appartenenti ai singoli rami della curva $\gamma^{900}$ che passano per $X$. Questo è anche d'accordo col fatto che un punto doppio uniplanare di una superficie si può considerare come un punto doppio ordinario (conico) a cui ne siano infinitamente vicini due altri in direzioni distinte (il che porta di conseguenza, in generale, che ve ne sia anche un terzo $\left.\left({ }^{*}\right)\right)$.

$\dot{\mathrm{E}}$ prevedibile altresì l'esistenza di un numero finito di spazi tritangenti, i quali incontrino $V$ secondo superficie con un punto biplanare e due punti conici (tutti distinti). Il loro numero verrà determinato fra poco (n. ${ }^{0} 20$.

19. Il numero $(=720$ ) degli spazi tritangenti con due punti di contatto infinitamente vicini, ossia il numero delle cuspidi della curva $0^{270}$ (del quale dal $n^{\circ} 12$ in poi non ci siamo mai valsi) può essere ora verificato nel modo che segue $\left(^{* *}\right)$

Consideriamo in $S_{4}$ un piano qualunque 5. Ogni $S_{3}$ passante per esso incontra la curva $\gamma^{10.99}$ in 10.90 punti; e lo spazio tangente a $V$ in uno qualunque di questi punti sarà tangente a $V$ stessa anche in altri due punti di quella curva, formanti col primo un trilatero di rette speciali. Facendo corrispondere a quel primo $S_{3}$ del fascio $\xi$ tutti quelli che dal medesimo piano $\xi$ proiettano questi ulteriori 2.10 .90 punti della curva $\%$ nasce nel fascio $\xi$ una corrispondenza simmetrica $(20.90,20.90)$, nella quale dovranno esistere 40.90 coincidenze. E queste coincidenze saranno di tre tipi diversi, perchè due $S_{3}$ omologhi possono coincidere: iettano;

1) senza che coincidano i due punti della linea $\gamma$ che essi rispett. pro-

(*) C. SEGRE, Sulla scomposizione dei punti singolari delle superficie algebriche (Annali di Nat., ser. 2 , vol. $25 ; 1896 ;$ n. ${ }^{\circ} 8$ ).

(**) Enriques, Mem. eit., p. 34-35. 
2) per il fatto che questi due punti sono sovrapposti, senza essere tuttavia infinitamente vicini sulla curva $\gamma$ : ossia cadono in un medesimo punto multiplo di questa curva;

3) per il fatto che vengono proprio a coincidere sulla curva $\gamma$ i due punti che quegli $S_{3}$ rispett. proiettano.

Nel primo caso la congiungente dei due punti considerati sulla linea $\%$ la quale è una retta speciale, dovrà appoggiarsi al piano $\%$ Ora a questo piano si appoggiano 90 rette speciali $p$ (generatrici di $\Sigma$ ), ciascuna delle quali incontra $\gamma$ in 28 punti (n. $\left.{ }^{\circ} 14\right)$; e corrispondentemente a ciascuno di questi 28 punti lo spazio proiettante $\xi$. p ha uno dei suoi omologhi che coincide con esso. Troviamo così 90 spazi del fascio $\zeta$, ciascuno dei quali assorbe 28 coincidenze; complessivamente dunque 28.90 coincidenze.

Il secondo caso si presenta soltanto per i 60 punti tripli della curva $\gamma$ considerati al n. ${ }^{0}$ prec. Lo spazio del fascio $\vdots$ che proietta uno di questi punti si può considerare in tre modi diversi come uno spazio di cui due omologhi coincidono con esso; esso assorbirà perciò 6 coincidenze.

Le coincidenze residue, in numero di :

$$
40.90-28.90-6.60=(40-28-4) .90=8.90=720
$$

saranno costituite dagli spazi del fascio $\xi$ che proiettano quei punti (semplici) di $\gamma$, ciascuno dei quali assorbe due vertici di un trilatero di rette speciali, ossia due punti di contatto (infinitamente vicini) di uno spazio tritangente. E queste sono coincidenze semplici. Sarà dunque questo stesso, ossia 720 , il numero degli spazi tritangenti con due punti di contatto infinitamente vicini; come già avevamo trovato precedentemente.

20. Le intersezioni della curva $\gamma^{10.30}$ colla varietà Hessiana di $V$ si ripartiranno fra quei punti di contatto degli spazi tritangenti, nei quali le superficie intersezioni di questi spazi con $V$ banno punti doppi biplanari (o uniplanari). Ciascuno di questi punti dovrà anche trovarsi sopra una almeno delle due curve $\mu^{30}$ e $\rho^{2 \% 0}$, che formano insieme l'intersezione della sviluppabile $\Sigma^{90}$, sulla quale sta $\gamma$, colla varietà Hessiana.

Anzitutto la superficie intersezione di $V$ con uno spazio tritangente può acquistare un punto doppio biplanare (del tipo $B_{4}$ ) per il fatto che due dei tre punti di contatto di questo spazio sono infinitamente vicini. Di questi punti biplanari ve ne sono $720=8.90$, e in ciascuno di essi la curva $\gamma$ è tangente alla retta asse del punto biplanare medesimo (n. ${ }^{0}$ 18), la quale a sua 
volta è ivi tangente alla Hessiana ( $\mathrm{n}^{\circ} 4$ ). Dunque ciascuno di questi punti assorbe due delle intersezioni cercate.

Gli spazi tangenti a $V$ nei 60 punti comuni ad essa e alla curva doppia della varietà Hessiana (sono particolari spazi tritangenti, e) incontrano $V$ secondo superficie con punto doppio uniplanare. Ciascuno di questi punti è triplo per la curva $\gamma\left(n .^{\circ} 18\right)$ e doppio per la Hessiana (senza che le tangenti a quella appartengano al cono tangente di questa), e assorbirà percid 6 intersezioni.

Tutte queste intersezioni appartengono alla linea $\mu^{90}$.

Le intersezioni residue, in numero di:

$$
5.10 .90-2.8 .90-6.60=30.90=2700
$$

dovranno cadere in punti di contatto di quegli spazi tritangenti pei quali $i$ tre punti di contatto son pur sempre distinti, ma uno di questi è per la superficie intersezione con $V$ punto doppio biplanare (in generale del tipo $B_{3}$ ). Queste intersezioni, che apparterranno alla linea $p^{270}$, saranno in generale intersezioni semplici. Vi saranno perciò, in generale, $30.90=2700$ spazi tritangenti che incontrano $V$ secondo superficie con un punto doppio biplanare $e$ due punti doppi conici. Questi due punti doppi conici apparterranno allo spigolo di regresso $\sigma^{270}$ della sviluppabile $\Sigma^{90}$.

21. Gli $\infty^{1}$ spazi tritangenti della varietà $V$ inviluppano un'altra superficie sviluppabile, anche proiettivamente legata a $V$, i cui piani tangenti e le cui generatrici saranno le intersezioni rispett. delle coppie e delle terne di spazi tritangenti consecutivi.

Uno generico $n$ fra questi spazi tritangenti toccherà $V$ in tre punti, congiunti a due a due da tre rette speciali $p, p^{\prime}, p^{\prime \prime}$; e lungo queste rette la varietà $V$ e la sviluppabile $\Sigma^{90}$ ammetteranno tre piani tangenti fissi $\pi, \pi^{\prime}, \pi^{\prime \prime}$, tutti contenuti nello spazio $\Pi$. Lo spazio tritangente $\mathrm{II}_{1}$ consecutivo a $\Pi$ conterrà tre piani analoghi $\pi_{1}, \pi^{\prime}, \pi^{\prime \prime}{ }_{1}$, rispett. consecutivi ai precedenti; $\mathrm{e}$ poichè le tre rette $p, p^{\prime}, p^{\prime \prime}$, generatrici di $\Sigma^{90}$, possono considerarsi rispett. come intersezioni delle coppie di piani tangenti consecutivi $\pi \pi_{1}, \pi^{\prime} \pi_{1}^{\prime}, \pi^{\prime \prime} \pi_{1}^{\prime \prime}$, cosi il piano $\Pi \Pi_{1}$ dovrà contenere queste stesse rette, e quindi i tre punti di contatto di II colla varietà $V$.

La sviluppabile inviluppata dagli $\infty^{1}$ spazi tritangenti di $V$ ha per piani tangenti $i$ piani determinati dalle terne di punti di contatto di questi medesimi spazi. 
Similmente, per trovare le generatrici di questa nuova sviluppabile, potremo osservare che le tre rette speciali $p, p^{\prime}, p^{\prime \prime}$ dianzi considerate si appoggiano rispett. alle tre consecutive $p_{1}, p_{i}^{\prime}, p^{\prime \prime}$, in altrettanti punti $P, P^{\prime}$, $P^{\prime \prime}$ appartenenti alla linea $\sigma^{270}$; e percio l'intersezione del piano $p p^{\prime} p^{\prime \prime}$ col piano consecutivo $p_{1} p_{1}^{\prime} p_{1}^{\prime \prime}$ dovrà contenere questi stessi tre punti, $i$ quali dovranno trovarsi pertanto in linea retta.

Le generatrici della stessa sviluppabile sono altrettante trisecanti della curva $\sigma^{2 \gamma n}$. Per agni trilatero di rette speciali i punti di contatto dei singoli lati colla curva $\sigma^{270}$ dovranno stare in linea retta; e le rette su cui stanno queste terne di punti saranno le generatrici della nuova sviluppabile (*).

22. Possiamo determinare facilmente l'ordine della nuova sviluppabile, e l'ordine della varietà dei suoi piani tangenti, considerandone le intersezioni colla varietà cubica $V$.

La varietà dei piani tangenti $p p^{\prime} p^{\prime \prime}$ incontra $V$ secondo la (sola) sviluppabile $\Sigma^{9 n}$, la quale va contata 14 volte, essendo questa la sua multiplicità per quella varietà di piani. L'ordine domandato sarà dunque

$$
\frac{14.90}{3}=14.30=420 \text {. }
$$

(*) Si puis verificare direttamente che per ogni trilatero di rette speciali i punti di contatto dei lati colla curva $\sigma^{270}$ stanno in linea retta. Infatti l'equazione di una varietic $Y$ della quale lo spazio $x_{0}=0$ sia uno spazio tritangente generico si puo mettere sotto la forma:

$$
x_{0} \gamma+x_{1}^{3}+\left(x_{2}+x_{3}+x_{4}\right) x_{1}^{2}+h x_{2} x_{3} x_{4}=0
$$

love $h$ è un coefliciente numerico, e $f \equiv \mathbf{\Sigma} a_{i k} x_{i} x_{k}$ (essendo i coefficienti $a_{92}, a_{33}, a_{44}$ tutti diversi da zero, se la varietà non ha punti dóppi). Lo spazio $x_{0}=0$ è allora tangente a $\checkmark$ nei tre punti fondamentali [2], [3]. [4], i quali saranno vertici di un trilatero di rette speciali (affatto generico). - Cercando le intersezioni del lato (ossia della retta speciale) $x_{0}=x_{1}=x_{2}=0$ colla varietà Hessiana di $V$ (a cui esso è bitangente), si trova che queste dipendono dall'equazione:

$$
\left(x_{3}+x_{4}\right)\left(a_{33} x_{3}^{2}-a_{44} x_{4}^{2}\right)^{2}=0
$$

di modo che sulla retta considerata sarà $x_{3}+x_{4}=0$ il punto indicato con $R$ al n. ${ }^{\circ} 12$, mentre i due punti $M, N$ saranno determinati dall'equazione $a_{39} a_{3}^{2}-a_{44} x_{4}^{2}=0$. Percio il punto di contatto della medesima retta colla linea $\sigma^{270}$, che è il coniugato armonico di $R$ rispetto alla coppia $M N$, saru definito dall'equazione $a_{38} x_{3}+a_{44} x_{4}=0$. Ed è chiaro che questo punto e i suoi analoghi sulle due rette $x_{0}=x_{1}=x_{3}=0$ e $x_{0}=x_{1}=x_{4}=0$ appartengono tutti alla retta:

$$
x_{0}=x_{1}=a_{22} x_{2}+a_{33} x_{3}+a_{44} x_{4}=0 .
$$


Quanto alla sviluppabile stessa, come superficie luogo di rette, la sua intersezione con $V$ si compone:

1. della curva $\sigma^{270}$, contata anche 14 rolte;

$2 .^{\circ}$ di un certo numero di generatrici, corrispondentemente a quei casi in cui la retta che contiene $\mathrm{i}$ punti di contatto di $\sigma^{2 \% 0}$ con un trilatero di rette speciali coincide con uno dei lati di questo stesso trilatero. Ciò avviene quando due dei tre lati toccano $\sigma^{270}$ nelle loro intersezioni col terzo lato, ossia per quei 2700 spazi tritangenti $\left(n .^{\circ} 20\right)$ che incontrano $V$ secondo superficie con un punto doppio biplanare e due punti doppi conici.

L'ordine della superficie sviluppabile di cui si tratta è dunque eguale a

$$
\frac{270.14+2700}{3}=270.8=2160
$$

La sviluppabile inviluppata dagli $\infty^{1}$ spazi tritangenti della variet⿳亠口 $V$ è di ordine 2160, e i suoi piani tangenti formano una varietà di ordine 420.

Questo inviluppo $\propto^{1}$ di spazi tritangenti la per elementi stazionari $i$ 2700 spazi che incontrano $V$ secondo superficie con un punto biplanare e due punti doppi conici: poichè le quadriche polari dei punti di uno di questi spazi hanno in ciascuno dei tre punti di contatto del medesimo spazio due intersezioni fisse colla curva $\gamma^{300}$. In uno di questi punti esse sono tangenti a $\gamma$, e gli altri due punti di contatto sono cuspidi di quest'ultima curva.

Di questo stesso inviluppo $\infty^{1}$ si può determinare anche il genere, con un'applicazione della formula di Zeuthen. Fra la sviluppabile $2^{90}$ e la varietà $\infty^{1}$ dei trilateri di rette speciali contenuti nei singoli spazi tritangenti si può stabilire una corrispondenza $(3,14)$, facendo corrispondere a ogni generatrice di $\Sigma^{90}$ i 14 trilateri a cui essa appartiene, e a ogni trilatero le 3 generatrici di $\Sigma^{90}$ che sono elementi. Fra i 14 trilateri che hanno per lato una data retta. speciale ve ne sono due coincidenti quando coincidono anche due dei 14 spazi tritangenti che passano per il piano $\pi$ tangente lungo quella retta; e questo spazio tritangente, che va contato due volte, non può essere che uno dej 2700 spazi già sopra considerati. D'altra parte vi sono 720 trilateri con due lati coincidenti (n. 18). Segue da ciò che nella formola generale:

$$
y-y^{\prime}=2 x\left(p^{\prime}-1\right)-2 x^{\prime}(p-1)
$$

si dovrà porre $x=3, x^{\prime}=14 ; y=2700, y^{\prime}=720$; e $p=136$. Si ricava allora $p^{\prime}=961$; e sarà questo il genere domandato. 
Tenendo conto dei diversi caratteri finora determinati e applicando lo solite formole (cfr. ad es. n. ${ }^{\circ} 11$ ), si trova che l'inviluppo degli spazi tritangenti ha uno spigolo di regresso di ordine 5820, con 11400 cuspidi.

Srazi Quadritangenti.

23. Dopo aver determinati gli $\infty^{2}$ spazi bitangenti della varietà $V$, abbiamo considerata (n.i $15-17$ ) la superfície $F^{7 \cdot 90}$ luogo di quei 14 poli di ognuno di questi spazi che sono distinti, in generale, dai due punti di contatto; e dall' intersezione di questa superficie con $V$, all'infuori delle linee $\mu^{90}$ e $\rho^{270}$, abbiamo ricavata la linea $\gamma^{000}$, luogo dei punti di contatto degli spazi tritangenti. In modo analogo potremo adesso considerare, corrispondentemente agli $\infty^{i}$ spazi tritangenti, la linea $\lambda$ luogo di quei 13 loro poli che sono in generale distinti dai punti di contatto; e fra le intersezioni di questa curva colla varieta $V$ dovranno trovarsi i punti di contatto degli spazi quadritangenti (*).

Determiniamo anzitutto l'ordine $x$ di questa linea $\lambda$. A tal uopo basterà osservare che una quadrica qualunque del sistema polare incontra la curva $\gamma^{10.90}$ in 20.90 punti, che si ripartiscono in $\frac{20.90}{3}$ terne di punti mutuamente coniugati nell' involuzione $I_{18}$. E le $2 x$ intersezioni della medesima quadrica colla curva $\lambda$ dovranno comporsi precisamente dei $\frac{20.90}{3}$ gruppi di 13 punti che insieme con quelle terne costituiscono altrettanti gruppi completi della $I_{16}$. Sarà dunque:

$$
2 x=13 . \frac{20.90}{3} \text { ossia } x=130.30 \text {. }
$$

Questa curva $\lambda^{130.30}$ starà sulla superficie $F^{7.90}$ considerata al n. ${ }^{0} 17$, e ne sarà anzi curva tripla. Infatti ogni punto di essa, essendo polo di uno spazio tritangente, apparterà alle $C_{3}^{7}$ residue di tre diverse rette speciali $p$ (formanti uno dei soliti trilateri); e al variare di quel punto sulla linea $\lambda$ queste $C_{3}^{\gamma}$ descriveranno tre falde della superficie $F^{7 \cdot 30}$, in generale distinte, passanti tutte per $\lambda$.

(*) Exriques, Mem. cit., p. 3.1-35. 
24. Vediamo ora come si distribuiscano le intersezioni della curva $\lambda^{130.30}$ colla varietà $V$. Si avranno di queste intersezioni ogni qual volta uno dei 13 poli ulterjori di uno spazio tritangente - dei quali poli la linea $\lambda$ è appurito il luogo - appartiene anch'esso alla varietà $V$ : e, quando ciò avviene, può darsi che questo polo ulteriore sia distinto dai primi tre, e allora esso sarà un quarto punto di contatto del medesimo spazio con $V$, e si avrà uno spazio quadritangente; ma può anche darsi che esso coincida con uno dei primi tre, ossia con uno dei tre punti di contatto dello spazio considerate, e allora questo punto di contatto, coincidendo con uno dei punti ad esso coniugati nell'involuzione $I_{16}$, starà sulla varietà Hessiana di $V^{\prime}$, e sarà un punto biplanáre o unjplanare per la superficie intersezione di $V$ collo spazio tritangente di cui si tratta.

Ora gli spazi tritangenti che incontrano $V$ secondo superficie con punti doppi biplanari o uniplanari sono:

I 30.90 spazi che determinano come sezioni superficie con due punti doppi conici e un punto biplanare del tipo $B_{3}\left(\mathrm{n}^{\circ} 20\right)$;

Gli 8.90 spazi che segano superficie con un punto doppio conico e un punto biplanare del tipo $B_{4}\left(\mathrm{n} .{ }^{\circ} 18\right)$;

I 60 spazi che segano superficie con punto uniplanare (n. ${ }^{\circ} 18$ ).

Nel primo caso due dei 16 poli dello spazio di cui si tratta coincidono nel punto biplanare della superficie intersezione di questo spazio con $V$; e perciò la linea $\lambda$ passerà anch'essa (semplicemente) per questo punto biplanare. Questo punto appartiene alla linea $\rho$, della quale è anzi punto doppio (poichè è punto $R$ - ofr. n. $^{\circ} 12$ - per ognuna delle due rette speciali uscenti da esso); dunque, delle tre falde della superficie $F^{7.00}$ che passano per esso, certo due sono osculatrici a $V$ in quel punto (che non è per esse punto singolare), e perciò la linea $\lambda$, che sta su di esse, avrà pure in quel punto un contatio di $2 .^{\circ}$ ordine con $V$. Ciascuno di questi 30.90 punti assorbirà dunque tre intersezioni (almeno) della linea $\lambda$ colla varietà $V$.

Nel secondo caso si tratta di uno spazio tritangente con due punti di contatto infinitamente vicini; e in questo punto di contatto coincidono allora non soltanto due, ma tre fra i 16 poli di quello spazio (*). Perciò questo spazio

(*) Più generalmente anzi, ogni spazio tangente il quale incontri $V$ secondo una superticie con un punto doppio biplanare del tipo $B_{4}$ ha tre dei suoi poli coincidenti in questo punto. Infatti questo punto $X$ apparterrà a una retta speciale $p$, sulla quale sara punto doppio dell'involuzione ivi segata dalle quadriche del sistema polare, e apparterrà 
conterrà anch'esso, complessivamente, quattro dei propri poli, e per il suo polo triplo passerà (semplicemente) la curva $\lambda$. In questo punto due delle tre falde della superficie $F^{7.90}$ si confondono (perchè nel triangolo dej punti di contatto sono venuti a coincidere due dei tre lati), ma la terza falda (quella che corrisponde al terzo lato del triangolo) è distinta da queste, passa semplicemente per quel punto, ed è ivi tangente a $V$ (perchè il punto di cui si tratta appartione alla sua linea di contatto $\mu$ con $V$ stessa). Perciò in tutti questi 720 punti la linea $\lambda$ sarà tangente (in generale semplicemente) alla varietà $V$.

Infire uno spazio tangente il quale incontri la varietà $V$ secondo una superficie con punto doppio uniplanare (del tjpo più generale) ha quattro dei suoi poli coincidenti nel punto di contatto: poichè le quadriche del sistema polare che passano per questo punto vi hanno un piano tangente fisso (lo stesso piano che è ivi tangente alla superficie cubica intersezione di $V$ collo spazio proposto), e hanno perciò, fuori di quel punto, sole 12 intersezioni. Dunque i 60 punti di questo tipo staranno anch'essi sulla curva $\lambda$. Dico ora che questa curva ha in ognuno di essi un contatto di $2 .^{\circ}$ ordine colla varietà $V$. Infatti la superficie $F^{\gamma \cdot 94}$ passa per ognuno di questi punti con tre falde completamente distinte; e per ciascuna di queste falde vengono ivi a riunirsi il contatto con $V$ lungo un ramo di linea $\mu$ e l'intersezione semplice con $V$ lungo un ramo di linea $\gamma\left({ }^{*}\right)$. Perciò ciascuna di queste falde sarà in quel punto

pure alla $C_{3}^{\mathbf{7}}$ residua di questa retta (v. la prima nota al n. ${ }^{0} 12$ ); perciò le $\boldsymbol{\infty}^{3}$ quadriche del sistema polare che passano per esso (e che sono le polari dei punti dello spazio tangrente considerato) saranno tutte ivi tangenti alla retta $p$, \& avranno a comune, fuori di quel punto, soltanto le 13 loro intersezioni residue colla eurva $C_{3}^{y}$. Lo spazio proposto avrà dunque soli 13 poli (tutti semplici) distinti da $X$, e perció gli altri tre coincideranno con $X$, c. s. V. d.

(*) L'equazione di una varietì cubica tangente allo spazio $x_{0}=0$ nel punto fondamentale [4] e incontrata da questo spazio secondo una superficie con punto uniplanare del tipo pit generale si puó mettere sotto la forma:

$x_{0} f+\left(x_{1}+x_{2}+x_{3}\right)^{2} x_{4}+x_{1} x_{2} x_{3}=0$
dove $f \equiv \stackrel{\aleph}{i k}_{0}^{4} x_{i} x_{k}$, e $x_{0}=x_{1}+x_{2}+x_{3}=0$ è il piano tangente a quest' ultima superficie nel punto uniplanare. I piani tangenti nel punto [4] alle tre falde della superficie $F^{7 \cdot 90}$ saranno allora rappresentati, nello spazio $x_{0}=0$, rispettivamente dalle equazioni:

$$
x_{1}=x_{2} \quad x_{2}=x_{3} \quad x_{3}=x_{1}
$$

e avranno a comune la retta $x_{1}=x_{2}=x_{3}$, che sara la tangente alla linea, $\lambda_{2}$ I tre rami della linea $\gamma$ saranno tangenti rispettivamente alla retta $x_{1}=x_{2}=0$ e alle due analoghe; e i.tre rami della linea $\mu$ alla retta $x_{1}-x_{2}=x_{3}=0$ e analoghe (ottenute permutando gli indici $1,2,3$ ). 
osculatrice alla varietà $V$; e lo stesso avverrà per la linea 2, comune alle tre fulde.

Si osservi poi che in nessuno dei punti finora considerati la linea $\lambda$ può avere con $V$, in generale, un contatto di ordine superiore a quello indicato, perchè ciò renderebbe il numero degli spazi quadritangenti inferiore a un limite, al di sotto del quale si potrebbe verificare - esaminando qualche caso particolare - ch' esso non può certamente discendere.

Le intersezioni residue della linea $i$ colla varietà $V$ saranno (precisamente) i punti di contatto degli spazi quadritangenti a $V$, i quali saranno punti tripli della sviluppabile $\Sigma^{9 !}$ e della sua curva doppia $\gamma^{10.00}$. Il loro numero sarà:

$$
\begin{aligned}
& 3.130 .30-3.30 .90-2.8 .90-3.60 \\
= & (130-90-16-2) .90=22.90=1980 .
\end{aligned}
$$

E il numero degli spazi quadritangenti sarà la quarta parte di quest'ultimo numero, cioè $11.45=495$.

La varietà cubica generale dello spazio $S_{4}$ ha 495 spazi quadritangenti.

E riassumendo:

Le rette speciali contenute in una varietà cubica generale formano una rigata svituppabile di ordine 90, con curva doppia di ordine 900, e 2040 $(=1980+60)$ punti tripli, che sono tali anche per la sux curva doppia.

Questa superficie sviluppabile e la sua curva doppia sono $i$ luoghi dei punti di contatto rispett. degli spazi bitangenti e degli spazi tritangenti della varietà cubica proposta.

Fra gli spazi tritangenti ve ne sono in particolare:

2700 che incontrano lu varietà secondo superficie con un punto doppio biplanare e due punti doppi conici;

r20 con due punti di contatlo infinitamente vicini;

60 coi tre punti di contatto riuniti in unico punto, che è punto uniplanare della superficie sezione;

495 quadritangenti (e perciò elementi quadrupli del sistema $\propto$ degli spazi tritangenti).

Queste ultime due categorie di spazi tocano la varietà nei

$$
60+4.495=2040
$$

punti che sono tripli per la sviluppabile sopra accennata e per la sua curva doppia. 
25. Il numero degli spazi quadritangenti di una varietà cubica di $S_{4}$ si può verificare direttamente. in qualche caso speciale; e ciò riesce particolarmente facile nel caso di una varietà con sei punti doppi indipendenti (e perciò generabile con tre reti proiettive di spazi $S_{3}$, in posizione generale $(*)$ ), avvertendo che dovranno allora considerarsi come quadritangenti tutti gli spazi che incontrano la varietà secondo superficie con 4 punti doppi (o casi particolari di queste), e che ogni spazio il quale contenga $h(\leq 4)$ punti doppi della varietà proposta e la tocchi in altri $4-h$ punti (semplici) dovrà contarsi come equivalente a $2^{h}$ spazi quadritangenti del caso generale (analogamente a ciò che avviene per le tangenti doppie delle linee piane, e i piani tritangenti delle superficie di $S_{3}$ ).

Cominciamo col dimostrare che una varietà cubica $V$ con sej punti doppi indipendenti non ha spazi quadritangenti propriamente detti, ossia non ammette sezioni iperpiane con 4 punti doppi indipendenti, dei quali nessuno sia doppio anche per essa. Infatti, se vi fosse uno spazio quadritangente non passante per alcun punto doppio di $V$, per ognuno dei suoi 4 punti di contatto le sei rette della varietà $V$ che ne escono $\left({ }^{* *}\right)$ dovrebbero coincidere a 2 a 2 ; e siccome le $\infty^{2}$ rette contenute in $V$ si ripartiscono in tre diversi sistemi, due di $1 .^{\circ}$ ordine e uno di $4 .^{\circ}$ ordine $\left({ }^{* *}\right)$, cosi una almeno di quelle tre rette dovrebbe essere comune a due di questi sistemi. Allora ogni spazio $S_{3}$ passante per questa retta comune $r$ incontrerà $V$ secondo una superficie cubica, sulla quale la $r$ conterà come 2 almeno fra le 27 rette ivi contenute; e perciò ognuna di queste $\boldsymbol{\infty}^{2}$ superficie sezioni dovrà avere sopra $r$ qualche punto doppio (****). Ora ciò non è possibile senza che la varietà $Y^{\prime}$ abbia anch'essa sopra $r$ almeno un punto doppio; e lo spazio quadritangente considerato passerebbe allora per questo punto doppio, contrariamente all'ipotesi che si era fatta.

26. Ogni spazio quadritangente della varietà proposta $V$ dovrà dunque passare per uno almeno dei sei punti doppi.

(*) C. SEGRE, Sulle varietá cubiche dello spazio a quattro dimensioni.... (Mem. Acc. di Torino, ser. II, vol. $39^{\circ}, 1888 ;$ n. $\left.{ }^{\circ} 13\right)$.

(**) E non è nemmeno possibile che da qualcuno di questi punti escano infinito rette contenute in $V$; perchè queste rette dovrebbero stare tutte nello spazio tangente in quel punto, e questo spazio non potrebbe allora incontrare $V^{r}$ secondo una superficie con 4 punti doppi.

(***) Stare, Meln, cit., n.. 1:3.

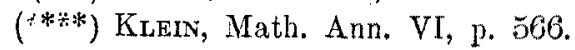


Ora in una varietà cubica con 6 punti doppi indipendenti il cono sestico di rette uscente da uno qualunque $X$ di questi punti si spezza in due coni cubici aventi a comune le cinque rette che congiungono $X$ medesimo agli altri cinque punti doppi; e proiettando la varietà dal punto doppio $X$ sopra uno spazio $S_{3} \equiv \pi$, quei due conici cubici vi determineranno come traccie due cubiche sghembe $k, k^{\prime}$ con cinque punti a comune (e contenute in una medesima quadrica). Tutte le superficie sezioni di $V$ con spazi $S_{3}$ passanti per $X$ si proietteranno sopra $\pi$ secondo piani; e in particolare quelle superficie che oltre ad $X$ hanno altri tre punti doppi indipendenti si proietteranno secondo piani tritangenti della curva complessiva formata dalle due cubiche $k$ e $k^{\prime}$, potendo tuttavia uno o più dei tre contatti venir sostituiti dal passaggio per altrettanti fra $i$ punti comuni alle stesse cubiche. A queste condizioni soddisfanno:

1) tutti i piani che congiungono tre dei cinque punti comuni alle cabiche $k$ e $k$, e questi saranno immagini di sezioni iperpiane passanti per $X$ e per tre altri punti doppi della varietà proposta;

2) tutti i piani che passano per uno dei punti comuni a quelle due cubiche, e sono tangenti a ciascuna di esse in un altro punto. Questi piani saranno immagini di sezioni determinate da spazi $S_{3}$ che passano per $X$ e per un secondo punto doppio, e toccano inoltre la varietà proposta in due punti ulteriori; e per ognuno dei punti comuni alle linee $k$ e $k^{\prime}$ passeranno quattro di questi piani (i piani tangenti comuni dei coni quadrici che da quel punto proiettano le due cubiche).

$\dot{\mathrm{E}}$ evidente poi che un piano tritangente della curva complessiva $k+k$ il quale passi per due dei 5 punti doppi non può a meno di passare anche per un terzo di questi punti; e non vi sono nemmeno piani tritangenti che non passino per.alcuno di questi punti doppi.

Riassumendo dunque, dovranno computarsi come spazi quadritangenti :

1) Tutti $\mathrm{i}\left(\begin{array}{l}6 \\ 4\end{array}\right)=15$ spazi che congiungono 4 fra i 6 punti doppi della varietà proposta, e ciascuno di questi sarà equivalente a 16 spazi quadritangenti propriamente detti;

2) Tutti gli spazi che passano per due di quei 6 punti doppi e toccano la varietà proposta in altri due punti (semplici), avvertendo che per ognuna delle $\left(\begin{array}{l}6 \\ 2\end{array}\right)=15$ coppie di punti doppi si possono condurre 4 di tali spazi, e che ognuno di essi va contato 4 volte. 
Questi spazi equivarranno complessivamente a

$$
16.15+4.4 .15=480
$$

spazi quadritangenti; nè vi saranno altre sezioni iperpiane con 4 punti doppi indipendenti.

Bisogna però ancora tener conto di quelle sezioni iperpiane che sono casi particolari di superficie con 4 punti doppi, potendosi ottenere da queste col far avvicinare indefinitamente due o più dei punti doppi medesimi. In particolare, facendo avvicinare indefinitamente due di questi punti, e contemporaneamente anche gli altri due (fra loro, ma non ai primi), la superficie si riduce al limite a una rigata cubica, avente per direttrice doppia la congiangente dei due punti doppi distinti che ancora rimangono (*). Ora ogni varietà cubica con due punti doppi ammette lungo la retta che congiunge questi due punti un $S_{3}$ tangente fisso, che l'incontra precisamente secondo una rigata cubica con questa stessa retta come direttrice doppia; e di queste sezioni iperpiane ve ne sono nel nostro caso $\left(\begin{array}{l}6 \\ 2\end{array}\right)$, ossia 15 . Nè vi sono altre sezioni jperpiane che debbano considerarsi come casi particolari di superficie con 4 punti doppi. Risulta perciò eguale a $480+15$, ossia 495 , il numero complessivo degli spazi quadritangenti, opportunamente valutati, come si era dimostrato in generale al n. 24 .

T'orino, Marzo 1901.

(*) Infatti l' equazione di una superficie cubica coi quattro punti doppi

$$
x=y=z=0, \quad x=y=w=0, \quad x=z=y+k w=0, \quad y=w=x+k^{\prime} z=0
$$

(dove $k$ e $k^{\prime}$ sono costanti non nulle) si può mettere sotto la forma:

$$
a\left(x+k^{\prime} z\right)(a y+b w)+y(y+k w)(c x+d z)=0
$$

dove $a, b, c, d$ sono le 4 costanti omogenee che devono ancora rimanere. Facendo avvicinare indefinitamente gli ultimi due punti doppi rispettivamente ai primi due, si ha al limite $k=k^{\prime}=0$; e l'equazione assume la forma:

$$
c^{2}(a y+b w)+y^{2}(c x+a z)=0
$$

che rappresenta precisamente una rigata colla direttrice doppia $x=y=0$. 